\title{
TOTAL VARIATION REGULARIZATION FOR IMAGE DENOISING, I. GEOMETRIC THEORY*
}

\author{
WILLIAM K. ALLARD ${ }^{\dagger}$
}

\begin{abstract}
Let $\Omega$ be an open subset of $\mathbf{R}^{n}$, where $2 \leq n \leq 7$; we assume $n \geq 2$ because the case $n=1$ has been treated elsewhere (see [S. S. Alliney, IEEE Trans. Signal Process., 40 (1992), pp. 1548-1562] and is quite different from the case $n>1$; we assume $n \leq 7$ because we will make use of the regularity theory for area minimizing hypersurfaces. Let $\mathcal{F}(\Omega)=\left\{f \in \mathbf{L}_{1}(\Omega) \cap \mathbf{L}_{\infty}(\Omega): f \geq 0\right\}$. Suppose $s \in \mathcal{F}(\Omega)$ and $\gamma: \mathbb{R} \rightarrow[0, \infty)$ is locally Lipschitzian, positive on $\mathbb{R} \sim\{0\}$, and zero at zero. Let $F(f)=\int_{\Omega} \gamma(f(x)-s(x)) d \mathcal{L}^{n} x$ for $f \in \mathcal{F}(\Omega)$; here $\mathcal{L}^{n}$ is Lebesgue measure on $\mathbb{R}^{n}$. Note that $F(f)=0$ if and only if $f(x)=s(x)$ for $\mathcal{L}^{n}$ almost all $x \in \mathbb{R}^{n}$. In the denoising literature $F$ would be called a fidelity in that it measures deviation from $s$, which could be a noisy grayscale image. Let $\epsilon>0$ and let $F_{\epsilon}(f)=\epsilon \mathbf{T V}(f)+F(f)$ for $f \in \mathcal{F}(\Omega)$; here $\mathbf{T V}(f)$ is the total variation of $f$. A minimizer of $F_{\epsilon}$ is called a total variation regularization of $s$. Rudin, Osher, and Fatemi and Chan and Esedoglu have studied total variation regularizations where $\gamma(y)=y^{2}$ and $\gamma(y)=|y|, y \in \mathbb{R}$, respectively. As these and other examples show, the geometry of a total variation regularization is quite sensitive to changes in $\gamma$. Let $f$ be a total variation regularization of $s$. The first main result of this paper is that the reduced boundaries of the sets $\{f>y\}, 0<y<\infty$, are embedded $C^{1, \mu}$ hypersurfaces for any $\mu \in(0,1)$ where $n>2$ and any $\mu \in(0,1]$ where $n=2$; moreover, the generalized mean curvature of the sets $\{f \geq y\}$ will be bounded in terms of $y, \epsilon$ and the magnitude of $|s|$ near the point in question. In fact, this result holds for a more general class of fidelities than those described above. A second result gives precise curvature information about the reduced boundary of $\{f>y\}$ in regions where $s$ is smooth, provided $F$ is convex. This curvature information will allow us to construct a number of interesting examples of total variation regularizations in this and in a subsequent paper. In addition, a number of other theorems about regularizations are proved.
\end{abstract}

Key words. total variation, regularization, image denoising

AMS subject classifications. 49Q20, 58E30

DOI. $10.1137 / 060662617$

1. Introduction and statement of main results. Throughout this paper, $n$ is an integer, $2 \leq n \leq 7, \Omega$ is an open subset of $\mathbb{R}^{n}$, and $\mathcal{L}^{n}$ is Lebesgue measure on $\mathbb{R}^{n}$.

We require $n \geq 2$ because the problems we consider are very different in case $n=1$; see [Alli]. We require $n \leq 7$ because we will be using the regularity theory of mass minimizing integral currents in $\mathbb{R}^{n}$ of codimension one; as is well known, these currents are free of interior singularities when $n \leq 7$ but may possess singularities if $n>7$; see [FE, sect. 5.4.15]. This work is motivated by image denoising applications in which it is often the case that $1 \leq n \leq 4$.

After a fairly lengthy discussion of results which occur in a setting more general than that of denoising, we treat denoising in section 1.8. See also sections 1.9, 8, and 10 as well as the examples in section 11 for more on denoising.

1.1. Some basic notation and conventions. Whenever $E \subset \Omega$ we frequently identify " $E$ " with " $1_{E}$," the indicator function of $E$.

The first appearance of any term which is about to be defined will always appear in italics or be displayed.

* Received by the editors June 9, 2006; accepted for publication (in revised form) April 20, 2007; published electronically DATE. This work was supported in part by Los Alamos National Laboratory. http://www.siam.org/journals/sima/x-x/66261.html

${ }^{\dagger}$ Department of Mathematics, Duke University, Durham, NC 27708-0320 (wka@math.duke.edu). 
We let

$$
\mathcal{F}(\Omega)=\left\{f \in \mathbf{L}_{1}(\Omega) \cap \mathbf{L}_{\infty}(\Omega): f \geq 0\right\}
$$

and

$$
\mathcal{M}(\Omega)=\left\{D: D \subset \Omega \text { and } 1_{D} \in \mathcal{F}(\Omega)\right\} ;
$$

thus a subset $D$ of $\Omega$ belongs to $\mathcal{M}(\Omega)$ if and only if $D$ is Lebesgue measurable and $\mathcal{L}^{n}(D)<\infty$. We endow $\mathbf{L}_{1}^{\text {loc }}(\Omega)$ with the topology induced by the seminorms $\mathbf{L}_{1}^{l o c}(\Omega) \ni f \mapsto \int_{K}|f| d \mathcal{L}^{n}$ corresponding to compact subsets $K$ of $\Omega$. Whenever $f \in \mathbf{L}_{1}^{\text {loc }}(\Omega)$ and $K$ is a compact subset of $\Omega$ we let

$$
\mathbf{k}(f, K)=\left\{g \in \mathbf{L}_{1}^{l o c}(\Omega): g(x)=f(x) \text { for } \mathcal{L}^{n} \text { almost all } x \in \Omega \sim K\right\} ;
$$

in other words, $g \in \mathbf{k}(f, K)$ if the support of the generalized function corresponding to $g-f$ is a subset of $K$. We let

$$
\mathbf{k}(f)=\cup\{\mathbf{k}(f, K): K \text { is a compact subset of } \Omega\} .
$$

Whenever $D$ is a Lebesgue measurable subset of $\Omega$ and $K$ is a compact subset of $\Omega$ we let

$$
\mathbf{k}(D, K)=\left\{E: E \subset \Omega \text { and } 1_{E} \in \mathbf{k}\left(1_{D}, K\right)\right\}
$$

and

$$
\mathbf{k}(D)=\cup\{\mathbf{k}(D, K): K \text { is a compact subset of } \Omega\} .
$$

Whenever $A, D, E$ are Lebesgue measurable subsets of $\Omega$ we let

$$
\Sigma_{A}(D, E)=\mathcal{L}^{n}(A \cap((D \sim E) \cup(E \sim D)))=\int_{A}\left|1_{D}-1_{E}\right| d \mathcal{L}^{n} ;
$$

note that $\mathcal{M}(\Omega) \times \mathcal{M}(\Omega) \ni(D, E) \mapsto \Sigma_{A}(D, E)$ is a pseudometric on $\mathcal{M}(\Omega)$.

Whenever $a \in \mathbb{R}^{n}$ and $0<r<\infty$ we let

$$
\mathbf{U}^{n}(a, r)=\left\{x \in \mathbb{R}^{n}:|x-a|<r\right\} \quad \text { and } \quad \mathbf{B}^{n}(a, r)=\left\{x \in \mathbb{R}^{n}:|x-a| \leq r\right\} .
$$

We let

$$
\text { int, cl, and bdry }
$$

stand for "interior," "closure," and "boundary," respectively, with respect to $\Omega$.

Whenever $A \subset \mathbb{R}^{n}$ and $a$ is an accumulation point of $A$ we let

$$
\operatorname{Tan}(A, a)=\bigcap_{0<r<\infty} \operatorname{cl}\left\{t(x-a): 0<t<\infty \text { and } x \in A \cap\left(\mathbf{B}^{n}(a, r) \sim\{a\}\right)\right\}
$$

and

$$
\operatorname{Nor}(A, a)=\bigcap_{w \in \operatorname{Tan}(A, a)}\left\{v \in \mathbb{R}^{n}: v \bullet w \leq 0\right\}
$$


Whenever $0 \leq m<\infty$ we let

$$
\mathcal{H}^{m}
$$

be an $m$-dimensional Hausdorff measure on $\mathbb{R}^{n}$.

We let

$$
\mathcal{X}(\Omega)
$$

be the space of smooth compactly supported vector fields on $\Omega$.

Whenever $y, z \in \mathbb{R}$ we let

$$
y \vee z=\max \{y, z\}, \quad y \wedge z=\min \{y, z\},
$$

and we note that $y+z=y \vee z+y \wedge z$.

1.2. Total variation. This work is based on the notion of the total variation of a locally summable function, which we now define.

Definition 1.1. Suppose $f \in \mathbf{L}_{1}^{\text {loc }}(\Omega)$. Then $\mathbf{T V}(f, \cdot)$, the total variation of $f$, is the largest Borel regular measure on $\Omega$ such that, for any open subset $U$ of $\Omega$,

$$
\mathbf{T V}(f, U)=\sup \left\{\int_{U} f \operatorname{div} X d \mathcal{L}^{n}: X \in \mathcal{X}(U) \text { and }|X| \leq 1\right\} .
$$

In particular, if $f$ is $C^{1}$ on $\Omega$ and $B$ is a Borel subset of $\Omega$, then

$$
\mathbf{T V}(f, B)=\int_{B}|\nabla f| d \mathcal{L}^{n} .
$$

Moreover, if $E$ a Lebesgue measurable subset of $\Omega$ with Lipschitz boundary, then $\mathbf{T V}(E, B)$ equals the $(n-1)$-dimensional Hausdorff measure of the intersection of the boundary of $E$ with $B$.

Suppose $f \in \mathbf{L}_{1}^{\text {loc }}(\Omega)$. We say $f$ is of bounded variation on $\Omega$ if $\mathbf{T V}(f, \Omega)$ is finite. If $\mathbf{T V}(f, \cdot)$ is a Radon measure on $\Omega$, which will be the case if and only if $\mathbf{T V}(f, K)<\infty$ whenever $K$ is a compact subset of $\Omega$, we say $f$ is of locally bounded variation on $\Omega$. We let

$$
\mathbf{B V}(\Omega) \text { and } \mathbf{B V}^{l o c}(\Omega)
$$

be the vector spaces of those $f \in \mathbf{L}_{1}(\Omega)$ which are of bounded variation on $\Omega$ and those $f \in \mathbf{L}_{1}^{\text {loc }}(\Omega)$ which are of locally bounded variation on $\Omega$, respectively.

If $E$ is a Lebesgue measurable subset of $\Omega$, the perimeter of $E$ is, by definition, $\mathbf{T V}(E, \Omega)$; we say $E$ is of locally finite perimeter if $E \in \mathbf{B V}^{l o c}(\Omega)$. As is well known, if $f \in \mathbf{B V}^{l o c}(\Omega)$, then $\{f>y\}$ is of locally finite perimeter for $\mathcal{L}^{1}$ almost all $y$. As is well known, sets of locally finite perimeter have nice rectifiability properties; see section 2.8 below.

\subsection{Total variation regularization.}

Definition 1.2. Suppose $F: \mathcal{F}(\Omega) \rightarrow \mathbb{R}$ and $0<\epsilon<\infty$. We let

$$
F_{\epsilon}: \mathcal{F}(\Omega) \rightarrow \mathbb{R} \cup\{\infty\},
$$

the total variation regularization of $F$ (with respect to $\epsilon$ ), be defined by setting

$$
F_{\epsilon}(g)=\epsilon \mathbf{T V}(g)+F(g) \quad \text { for } g \in \mathcal{F}(\Omega) .
$$


We let

$$
\mathbf{m}_{\epsilon}^{\text {loc }}(F)=\left\{f \in \mathcal{F}(\Omega) \cap \mathbf{B V}(\Omega): F_{\epsilon}(f) \leq F_{\epsilon}(g) \text { whenever } g \in \mathcal{F}(\Omega) \cap \mathbf{k}(f)\right\} .
$$

All of the statements and proofs of this paper, after straightforward modification, go through with the condition " $f \geq 0$," omitted in the definition of $\mathcal{F}(\Omega)$; however, the modified statements and proofs often break into two cases because if $f \in \mathbf{L}_{1}(\Omega)$ and $y \in \mathbb{R}$, one can only be sure that $\mathcal{L}^{n}(\{f>y\})<\infty$ if $y>0$ and that $\mathcal{L}^{n}(\{f<y\})<\infty$ if $y<0$.

It will be useful to extend the foregoing notions to functionals defined on sets, as follows.

Definition 1.3. Suppose $M: \mathcal{M}(\Omega) \rightarrow \mathbb{R}$ and $0<\epsilon<\infty$. We let

$$
M_{\epsilon}: \mathcal{M}(\Omega) \rightarrow \mathbb{R} \cup\{\infty\},
$$

the total variation regularization of $M$ (with respect to $\epsilon$ ), be defined by setting

$$
M_{\epsilon}(E)=\epsilon \mathbf{T V}(E)+M(E) \quad \text { for } E \in \mathcal{M}(\Omega) .
$$

We let

$$
\mathbf{n}_{\epsilon}^{\text {loc }}(M)=\left\{D \in \mathcal{M}(\Omega) \cap \mathbf{B V}(\Omega): M_{\epsilon}(D) \leq M_{\epsilon}(E) \text { whenever } E \in \mathcal{M}(\Omega) \cap \mathbf{k}(D)\right\} \text {. }
$$

The main purpose of this paper is to study the geometry and regularity of the sets $\{f>y\}$ and $\{f \geq y\}, y \in(0, \infty)$, when $f \in \mathbf{m}_{\epsilon}^{l o c}(F)$, provided $F$ satisfies certain conditions which we now describe. We will relate these results to certain methods for image denoising.

\subsection{Admissibility. Suppose}

$$
F: \mathcal{F}(\Omega) \rightarrow \mathbb{R}
$$

All our results will require $F$ to be admissible, a notion we now define.

Definition 1.4. We say $F$ is admissible if the restriction of $F$ to any subset of $\mathcal{F}(\Omega)$ which is bounded with respect to $\|\cdot\|_{\mathbf{L}_{\infty}(\Omega)}$ is Lipschitz with respect to $\|\cdot\|_{\mathbf{L}_{1}(\Omega)}$.

In other words, $F$ is admissible if whenever $0<Y<\infty$ we have

$$
\mathrm{l}(F, Y)<\infty
$$

where $\mathbf{l}(F, Y)$ is the infimum of the set of $L \in(0, \infty)$ such that

$$
|F(f)-F(g)| \leq L \int_{\Omega}|f-g| d \mathcal{L}^{n}
$$

whenever $f, g \in \mathcal{F}(\Omega)$ and $\max \left\{\|f\|_{\mathbf{L}_{\infty}(\Omega)}, \mid g \|_{\mathbf{L}_{\infty}(\Omega)}\right\} \leq Y$.

The notion of admissibility extends naturally to functionals on sets, as follows.

Definition 1.5. Suppose $M: \mathcal{M}(\Omega) \rightarrow \mathbb{R}$. We let

$$
\mathbf{l}(M)
$$

be the infimum of the set of $L \in(0, \infty)$ such that

$$
|M(D)-M(E)| \leq L \Sigma_{\Omega}(D, E) \quad \text { whenever } D, E \in \mathcal{M}(\Omega) .
$$

We say $M$ is admissible if $\mathbf{l}(M)<\infty$. 
1.5. $\mathcal{B}_{\lambda}(\Omega)$ and $\mathcal{C}_{\lambda}(\Omega)$. These spaces will be indispensable in this work.

Definition 1.6. Suppose $0 \leq \lambda<\infty$. We let

$$
\mathcal{B}_{\lambda}(\Omega)
$$

be the set of those $f \in \mathbf{B V}^{\text {loc }}(\Omega)$ such that for each compact subset $K$ of $\Omega$ we have

$$
\mathbf{T V}(f, K) \leq \mathbf{T V}(g, K)+\lambda \int_{\Omega}|f-g| d \mathcal{L}^{n} \quad \text { whenever } g \in \mathbf{k}(f, K) .
$$

We let

$$
\mathcal{C}_{\lambda}(\Omega)
$$

be the set of those Lebesgue measurable subsets $D$ of $\Omega$ with locally finite perimeter such that for each compact subset $K$ we have

$$
\mathbf{T V}(D, K) \leq \mathbf{T V}(E, K)+\lambda \Sigma_{\Omega}(D, E) \quad \text { whenever } E \in \mathbf{k}(D, K)
$$

The following result is based on ideas found in [BDG].

Theorem 1.1. Suppose $0 \leq \lambda<\infty$ and $f \in \mathbf{B V}^{l o c}(\Omega)$. Then

$$
f \in \mathcal{B}_{\lambda}(\Omega) \Rightarrow\{f>y\} \in \mathcal{C}_{\lambda}(\Omega) \quad \text { for } y \in \mathbb{R} .
$$

Conversely, if $D$ is a dense subset of $\mathbb{R}$, then

$$
\{f>y\} \in \mathcal{C}_{\lambda}(\Omega) \text { for } y \in D \Rightarrow f \in \mathcal{B}_{\lambda}(\Omega) .
$$

An immediate corollary is that $D \in \mathcal{C}_{\lambda}(\Omega)$ if and only if $1_{D} \in \mathcal{B}_{\lambda}(\Omega)$.

More results on $\mathcal{C}_{\lambda}(\Omega)$ and $\mathcal{B}_{\lambda}(\Omega)$ may be found in section 5.1.

1.5.1. The regularity theorem for $\mathcal{C}_{\lambda}(\Omega)$. The proof of the following theorem is an exercise, carried out in section 5.4, in the use of techniques from geometric measure theory which have been in the literature for over thirty years.

Note that in the following theorem, $\theta$ does not depend on $D$.

Theorem 1.2 (regularity). Suppose $0<\mu<\infty$ and $0<\beta<1$. There exists $\theta$ such that $0<\theta<1$ and with the following property:

Suppose

(i) $a \in \mathbb{R}^{n}$ and $0<R<\infty$;

(ii) $0 \leq \lambda<\infty, \lambda R \leq \theta$, and $D \in \mathcal{C}_{\lambda}\left(\mathbf{U}^{n}(a, R)\right)$;

(iii) $\Gamma$ is the interior of the support of the generalized function corresponding to the indicator function of $D$ and $M$ is the boundary of $\Gamma$ relative to $\mathbf{U}^{n}(a, R)$.

Then $\Sigma_{\mathbf{U}^{n}(a, R)}(D, \Gamma)=0$ and $M$ is an embedded hypersurface in $\mathbf{U}^{n}(a, R)$ of class $C^{1, \mu}$; moreover, if $N$ is a continuous field of unit normals to $M$ and $r=\theta R$, then

$$
|N(x)-N(w)| \leq \beta(|x-w| / r)^{\mu} \quad \text { whenever } x, w \in M \cap \mathbf{U}^{n}(a, r) ;
$$

finally, if $L$ is a line perpendicular to $\operatorname{Tan}(M, a)$, then $L$ intersects $M \cap \mathbf{U}^{n}(a, r)$ in at most one point.

In case $n=2$ we may take $\mu=1$. 
1.5.2. The relationship between admissibility and $\mathcal{C}_{\lambda}(\Omega)$. The following simple proposition relates the notion of admissibility to the spaces $\mathcal{B}_{\lambda}(\Omega)$.

Proposition 1.1. Suppose $F: \mathcal{F}(\Omega) \rightarrow \mathbb{R}, F$ is admissible, $0<\epsilon<\infty$, $f \in \mathbf{m}_{\epsilon}^{l o c}(F), Y=\|f\|_{\mathbf{L}_{\infty}(\Omega)}$, and $\lambda=\mathbf{l}(F, Y) / \epsilon$.

Then $f \in \mathcal{B}_{\lambda}(\Omega)$.

Proof. Suppose $g \in \mathbf{k}(f, K)$. Let $h=(g \wedge Y) \vee 0$. Then $h \in \mathbf{k}(f, K)$, so

$$
\epsilon(\mathbf{T V}(f, K)-\mathbf{T V}(h, K)) \leq F(f)-F(h) \leq \mathbf{l}(F, Y) \int_{\Omega}|f-h| .
$$

As is well known and shown in Proposition 2.3 below, $\mathbf{T V}(h, K) \leq \mathbf{T V}(g, K)$, and it is evident that $\int_{\Omega}|f-h| d \mathcal{L}^{n} \leq \int_{\Omega}|f-g| d \mathcal{L}^{n}$, so the proposition is proved.

We leave the even simpler proof of the following proposition to the reader.

Proposition 1.2. Suppose $M: \mathcal{M}(\Omega) \rightarrow \mathbb{R}, M$ is admissible, $0<\epsilon<\infty$, $D \in \mathbf{n}_{\epsilon}^{\text {loc }}(M)$, and $\lambda=\mathbf{l}(M) / \epsilon$.

Then $D \in \mathcal{C}_{\lambda}(\Omega)$.

Remark 1.1. Thus if $f \in \mathbf{m}_{\epsilon}^{\text {loc }}(F)$, where $F$ is admissible, the regularity theorem, Theorem 1.2, for $\mathcal{C}_{\lambda}(\Omega)$ applies to the sets $\{f>y\}, 0<y<\infty$. In particular, if $n>2$ and $0<\mu<1$ or if $n=2$ and $0<\mu \leq 1$, the boundaries of the support of $[\{f>y\}], 0<y<\infty$, are always embedded $C^{1, \mu}$ hypersurfaces.

In order to obtain yet more information about $\{f>y\}$ we need to assume more about $F$, as follows.

\subsection{Locality.}

Definition 1.7. Suppose $F: \mathcal{F}(\Omega) \rightarrow \mathbb{R}$. We say $F$ is local if $F$ is admissible and

$$
\hat{F}(f+g)=\hat{F}(f)+\hat{F}(g) \text { whenever } f, g \in \mathcal{F}(\Omega) \text { and } f g=0,
$$

where we have set

$$
\hat{F}(f)=F(f)-F(0) \quad \text { for } f \in \mathcal{F}(\Omega) .
$$

The notion of locality extends naturally to functionals on sets, as follows.

Definition 1.8. Suppose $M: \mathcal{M}(\Omega) \rightarrow \mathbb{R}$. We say $M$ is local if $M$ is admissible and

$$
\hat{M}(D \cup E)=\hat{M}(D)+\hat{M}(E) \quad \text { whenever } D, E \in \mathcal{M}(\Omega) \text { and } D \cap E=\emptyset,
$$

where we have set

$$
\hat{M}(E)=M(E)-M(\emptyset) \quad \text { for } E \in \mathcal{M}(\Omega) .
$$

The proofs of the following four propositions, which we carry out in section 6 , are exercises in real variable theory.

Proposition 1.3. Suppose $M: \mathcal{M}(\Omega) \rightarrow \mathbb{R}, M$ is admissible, and

$$
\mu(x)=\limsup _{r \downarrow 0} \frac{\hat{M}\left(\mathbf{B}^{n}(x, r)\right)}{\mathcal{L}^{n}\left(\mathbf{B}^{n}(x, r)\right)} \quad \text { for } x \in \Omega .
$$

Then $M$ is local if and only if

$$
M(E)=M(\phi)+\int_{E} m d \mathcal{L}^{n} \quad \text { for } E \in \mathcal{M}(\Omega)
$$


for some bounded Borel function $m: \Omega \rightarrow \mathbb{R}$, in which case $m(x)=\mu(x)$ for $\mathcal{L}^{n}$ almost all $x \in \Omega$.

Proposition 1.4. Suppose $F: \mathcal{F}(\Omega) \rightarrow \mathbb{R}, F$ is admissible, and

$$
\kappa(x, y)=\limsup _{r \downarrow 0} \frac{\hat{F}\left(y 1_{\mathbf{B}^{n}(x, r)}\right)}{\mathcal{L}^{n}\left(\mathbf{B}^{n}(x, r)\right)} \quad \text { for }(x, y) \in \Omega \times[0, \infty) .
$$

Then $F$ is local if and only if

$$
F(f)=F(0)+\int_{\Omega} k(x, f(x)) d \mathcal{L}^{n} x \quad \text { whenever } f \in \mathcal{F}(\Omega)
$$

for some Borel function $k: \Omega \times[0, \infty) \rightarrow \mathbb{R}$ such that

(i) $k(x, 0)=0$ for $\mathcal{L}^{n}$ almost all $x \in \Omega$;

(ii) whenever $0<Y<\infty$ there is $L \in[0, \infty)$ such that if $0 \leq y<z<Y$, then

$$
|k(x, y)-k(x, z)| \leq L|y-z| \quad \text { for } \mathcal{L}^{n} \text { almost all } x \in \Omega,
$$

in which case, for each $y \in[0, \infty), k(x, y)=\kappa(x, y)$ for $\mathcal{L}^{n}$ almost $x \in \Omega$.

Remark 1.2. Suppose $\beta: \mathbb{R} \rightarrow \mathbb{R}$ is locally Lipschitzian and

$$
F(f)=\beta\left(\int_{\Omega} f d \mathcal{L}^{n}\right) \quad \text { for } f \in \mathcal{F}(\Omega) .
$$

It is a evident that $F$ is admissible but not local unless $\beta$ is an affine function.

Perhaps a more interesting example is as follows. Suppose $K, s \in \mathbf{L}_{1}\left(\mathbb{R}^{n}\right)$ and

$$
F(f)=\int_{\mathbb{R}^{n}}|K * f-s| d \mathcal{L}^{n} \quad \text { for } f \in \mathcal{F}\left(\mathbb{R}^{n}\right) .
$$

Evidently, $\mathbf{l}(F, Y)=\|K\|_{\mathbf{L}_{1}\left(\mathbb{R}^{n}\right)}<\infty$ whenever $0<Y<\infty$, so $F$ is admissible. If $\kappa$ is as in Proposition 1.4, we find that $\kappa(x, y)=|K(x) y-s(x)|$ for $\mathcal{L}^{n}$ almost all $x$ and all $y \in(0, \infty)$. It is easy to see that $F$ is not local if both $\{K>0\}$ and $\{K<0\}$ have positive Lebesgue measure.

Proposition 1.5. Suppose $F: \mathcal{F}(\Omega) \rightarrow \mathbb{R}, F$ is admissible, $\kappa$ is as in Proposition 1.4 ,

$$
u(x, y)=\limsup _{z \rightarrow y} \frac{\kappa(x, z)-\kappa(x, y)}{z-y} \quad \text { for }(x, y) \in \Omega \times(0, \infty),
$$

and, for each $y \in(0, \infty)$,

$$
U_{y}(E)=\limsup _{z \rightarrow y} \frac{F\left(z 1_{E}\right)-F\left(y 1_{E}\right)}{z-y} \quad \text { for } E \in \mathcal{M}(\Omega) .
$$

Then

(i) $u$ is a Borel function and $|u(x, y)| \leq l(F, Y)$ whenever $x \in \Omega$ and $0<y<$ $Y<\infty$;

(ii) $\left|U_{y}(E)\right| \leq l(F, Y) \mathcal{L}^{n}(E)$ whenever $0<y<Y$ and $E \in \mathcal{M}(\Omega)$;

(iii) for any $f \in \mathcal{F}(\Omega),(0, \infty) \ni y \mapsto U_{y}(\{f>y\})$ is a Borel function.

Moreover, if $F$ is local, then 
(iv) for $\mathcal{L}^{1}$ almost all $y \in(0, \infty), U_{y}$ is local, $\mathbf{l}\left(U_{y}\right) \leq \mathbf{l}(F, Y)$ whenever $y<Y<$ $\infty$, and

$$
U_{y}(E)=\int_{E} u(x, y) d \mathcal{L}^{n} x \quad \text { whenever } E \in \mathcal{M}(\Omega) ;
$$

(v) for any $f \in \mathcal{F}(\Omega)$,

$$
F(f)=F(0)+\int_{0}^{\infty} U_{y}(\{f>y\}) d \mathcal{L}^{1} y .
$$

Proposition 1.6. Suppose $F: \mathcal{F}(\Omega) \rightarrow \mathbb{R}, F$ is local, $\kappa$ is as in Proposition 1.4, and $U_{y}, 0<y<\infty$, and $u$ are as in Proposition 1.5 .

The following are equivalent:

(i) $F$ is convex.

(ii) $\mathbb{R} \ni y \mapsto F\left(y 1_{E}\right)$ is convex for any $E \in \mathcal{M}(\Omega)$.

(iii) For any $x \in \Omega$,

$$
\mathbb{R} \ni y \mapsto \kappa(x, y) \text { is convex. }
$$

(iv) $\mathbb{R} \ni y \mapsto U_{y}(E)$ is nondecreasing for any $E \in \mathcal{M}(\Omega)$.

(v) For any $x \in \Omega$,

$$
\mathbb{R} \ni y \mapsto u(x, y) \text { is nondecreasing. }
$$

Moreover, if $F$ is convex and $0<y<\infty$, then $U_{y}$ is local and

$$
\lim _{z \downarrow y} U_{z}(E)=U_{y}(E) \quad \text { whenever } E \in \mathcal{F}(\Omega) .
$$

Remark 1.3. Suppose $F: \mathcal{F}(\Omega) \rightarrow \mathbb{R}, F$ is admissible, $\kappa$ is as in Proposition 1.4,

$$
l(x, y)=\liminf _{z \rightarrow y} \frac{\kappa(x, z)-\kappa(x, y)}{z-y} \text { for }(x, y) \in \Omega \times(0, \infty),
$$

and, for each $y \in(0, \infty)$,

$$
L_{y}(E)=\liminf _{z \rightarrow y} \frac{F\left(z 1_{E}\right)-F\left(y 1_{E}\right)}{z-y} \text { for } E \in \mathcal{M}(\Omega) .
$$

Modifying the proof of Proposition 1.5 in a straightforward way one finds that this proposition holds with $u$ and $U_{y}, 0<y<\infty$, replaced by $l$ and $L_{y}, 0<y<\infty$, respectively. Evidently, for any $E \in \mathcal{M}(\Omega)$ we have

$$
L_{y}(E) \leq U_{y}(E) \quad \text { whenever } 0<y<\infty
$$

with equality for $\mathcal{L}^{1}$ almost all $y \in(0, \infty)$.

Moreover, if $F$ is local, one finds that by modifying the proof of Proposition 1.6 in a straightforward way that this proposition holds with $u$ and $U_{y}, 0<y<\infty$, replaced by $l$ and $L_{y}, 0<y<\infty$, respectively, except that (1.4) must be replaced by

$$
\lim _{z \uparrow y} L_{z}(E)=L_{y}(E) \quad \text { whenever } E \in \mathcal{F}(\Omega) .
$$

We will show at the end of section 6 that for all but countably many $y \in(0, \infty)$

$$
L_{y}(E)=U_{y}(E) \quad \text { whenever } E \in \mathcal{M}(\Omega) .
$$

See section 1.9 for a natural example where $L_{y} \neq U_{y}$ for some $y \in(0, \infty)$. 
1.6.1. When $\boldsymbol{F}$ is local and convex. Things get a lot more interesting when $F$ is local and convex. An important result, which will be proved in section 6 , is the following.

TheOrem 1.3. Suppose $F: \mathcal{F}(\Omega) \rightarrow \mathbb{R}, F$ is local and convex, and $f \in \mathbf{m}_{\epsilon}^{\text {loc }}(F)$. Then

$$
\{f \geq y\} \in \mathbf{n}_{\epsilon}^{l o c}\left(L_{y}\right) \quad \text { and } \quad\{f>y\} \in \mathbf{n}_{\epsilon}^{l o c}\left(U_{y}\right) \quad \text { whenever } 0<y<\infty .
$$

When I wrote the initial version of this paper I thought this theorem was completely new. I was wrong. As a referee has pointed out, essentially the following result appears as Proposition 2.2 of [CA2].

Suppose $s \in \mathcal{F}(\Omega)$,

$$
F(g)=\frac{1}{2} \int_{\Omega}|g-s|^{2} d \mathcal{L}^{2} \quad \text { for } g \in \mathbf{L}_{2}(\Omega)
$$

$0<\epsilon<\infty$, and $f \in \mathbf{L}_{2}(\Omega)$ is such that

$$
\epsilon \mathbf{T V}(f, \Omega)+F(f) \leq \epsilon \mathbf{T V}(g, \Omega)+F(g) \quad \text { for } g \in \mathbf{L}_{2}(\Omega) .
$$

Then $f(x) \geq 0$ for $\mathcal{L}^{n}$ almost all $x \in \Omega$ and, whenever $0<y<\infty$,

$$
\epsilon \mathbf{T V}(\{f>y\})+U_{y}(\{f>y\}) \leq \epsilon \mathbf{T V}(E)+U_{y}(E) \quad \text { for } E \in \mathcal{M}(\Omega),
$$

where $U_{y}(E)=\int_{E} y-s d \mathcal{L}^{n}$ for $E \in \mathcal{M}(\Omega)$.

Note that $f$ above is a global minimizer of $\mathbf{L}_{2}(\Omega) \ni g \mapsto \epsilon \mathbf{T V}(g, \Omega)+F(g)$. In fact, the method used to prove this result in [CA2] can be extended to a very general class of local and convex $F$ 's but still for global minimizers. For example, I do not see how to apply this method to the case when $\Omega$ has Lipschitz boundary and one minimizes in the class of $f$ 's with a given trace on the boundary of $\Omega$, a situation in which Theorem 1.3 clearly applies.

The following theorem, which will be proved in section 6 , is more than a converse of the preceding theorem. This result is of particular interest when $\gamma(y)=|y|$ for $y \in \mathbb{R}$ in section 1.8; it is the essential ingredient in the proof of Theorem 1.7.

TheOREm 1.4. Suppose $F: \mathcal{F}(\Omega) \rightarrow \mathbb{R}, F$ is local and convex, $G$ is a $\mathcal{L}^{n} \times \mathcal{L}^{1}$ measurable subset of $\Omega \times(0, \infty)$ such that

(i) $\left(\mathcal{L}^{n} \times \mathcal{L}^{1}\right)(G)<\infty$;

(ii) $\{y \in(0, \infty):\{(x, y) \in G\} \neq \emptyset\}$ is bounded;

(iii) $\{x:(x, y) \in G\} \in \mathbf{n}_{\epsilon}^{\text {loc }}\left(U_{y}\right)$ for $\mathcal{L}^{1}$ almost all $y \in(0, \infty)$, and $f: \Omega \rightarrow[0, \infty)$ is such that

$$
f(x)=\mathcal{L}^{1}\{y:(x, y) \in G\} \quad \text { for } x \in \Omega
$$

Then $f \in \mathbf{m}_{\epsilon}^{\text {loc }}(F)$.

It turns out that a set $G$ as in the previous theorem is essentially unique, provided $F$ is strictly convex. Simple examples in [CE] show this is not the case if $F$ is merely convex. Bear in mind that $f$ below is essentially unique because $F$ is strictly convex.

TheOrem 1.5. Suppose $F, G$, and $f$ are as in the preceding theorem and $F$ is strictly convex. Then

$$
\left(\mathcal{L}^{n} \times \mathcal{L}^{1}\right)((G \sim\{(x, y): f(x)>y>0\}) \cup(\{(x, y): f(x)>y>0\} \sim G))=0 .
$$

See section 9.3 for the proof. 
1.7. Results on curvature. A good deal of the following theorem, which will be proved in section 7, is well known. If one assumes that $M$ below is of class $C^{2}$, the formula for $H$ in (1.7) may be derived by a straightforward variational argument which appears in $[\mathrm{M}]$; in our case, in the light of the regularity theorem for $\mathcal{C}_{\lambda}(\Omega)$ we know only that $M$ is of class $C^{1, \mu}, 0<\mu<1$, so one must proceed a bit more carefully. We represent $M$ locally as a graph of a function which satisfies an elliptic equation and appeal to higher regularity results for such equations as appear, for example, in [GT]. One may then obtain the second variation formula (1.8) which, obviously, is a global constraint on a member of $\mathbf{n}_{\epsilon}^{\text {loc }}(Z)$ to which it applies. I believe (1.8) is new; it will be used in section 11 and [AW2] when we construct minimizers.

See section 4 for the definitions of mean curvature and second fundamental form which we use.

THEOREM 1.6. Suppose

(i) $\zeta \in \mathbf{L}_{\infty}(\Omega)$ and $Z(E)=\int_{E} \zeta d \mathcal{L}^{n}$ whenever $E \in \mathcal{M}(\Omega)$;

(ii) $U$ is an open subset of $\Omega, j$ is a nonnegative integer, $0<\mu<1$, and $\zeta \mid U$ is of class $C^{j, \mu}$;

(iii) $0<\epsilon<\infty$ and $D \in \mathbf{n}_{\epsilon}^{\text {loc }}(Z)$;

(iv) $\Gamma$ is the intersection of $U$ with the interior of the support of the generalized function corresponding to the indicator function of $D$ and $M$ is the boundary of $\Gamma$ relative to $U$.

Then $\Sigma_{U}(D, \Gamma)=0, M$ is an embedded hypersurface of class $C^{j+2, \mu}$, and

$$
H(x)=-\frac{1}{\epsilon} \zeta(x) \nu(x) \quad \text { for } x \in M,
$$

where $H$ is the mean curvature vector of $M$ and $\nu$ is the unit normal along $M$ which points out of $\Gamma$.

Moreover, if $\zeta$ is of class $C^{1}$ on $U$ and $Q$ is the square of the length of the second fundamental form of $M$, then

$$
\int_{M} \epsilon\left(\left|\nabla_{M} \phi(x)\right|^{2}-\phi(x)^{2} Q(x)\right)-\phi(x)^{2} \nabla \zeta(x) \bullet \nu(x) d \mathcal{H}^{n-1} x \geq 0
$$

for any smooth compactly supported function $\phi$ on $U$; here, for each $x \in M, \nabla_{M} \phi(x)$ is the orthogonal projection of $\nabla \phi(x)$ on $\operatorname{Tan}(M, x)$.

In case $n=2$ we may take $\mu=1$.

This theorem will apply in the context of denoising if $s$ as in section 1.8 is sufficiently regular in $U$.

1.8. Denoising. Suppose

(i) $s \in \mathcal{F}(\Omega)$;

(ii) $\gamma: \mathbb{R} \rightarrow[0, \infty), \gamma$ is locally Lipschitzian, $\gamma$ is decreasing on $(-\infty, 0), \gamma(0)=0$, and $\gamma$ is increasing on $(0, \infty)$;

(iii) $F(f)=\int_{\Omega} \gamma(f(x)-s(x)) d \mathcal{L}^{n} x$ for $f \in \mathcal{F}(\Omega)$.

Here $s$ could be a grayscale representation of a degraded image which we wish to denoise. In the context of denoising $F$ would be called a fidelity; it is a measure of how much $f$ differs from $s$. If $0<\epsilon<\infty$, the members of $\mathbf{m}_{\epsilon}^{\text {loc }}(F)$ would be called total variation regularizations of $s$ (with respect to the fidelity $F$ and smoothing parameter $\epsilon$ ).

In the literature $F_{\epsilon}$ is often replaced by $F_{\epsilon} / \epsilon$ and $\lambda=1 / \epsilon$ is thought of as a Lagrange multiplier. 
For a very informative discussion of the use of total variation regularizations in the field of image processing, see the introduction of [CE]. We will not discuss image processing any further except to note that the notion of total variation regularization in image processing is useful for other purposes besides denoising.

Evidently, $F$ is admissible, so Proposition 1.1 holds and the results of section 1.5 apply. It is also evident that $F$ is local.

Let us now assume $\gamma$ is convex. It follows that $F$ is convex. Set

$$
\alpha(y)=\liminf _{z \rightarrow y} \frac{\gamma(z)-\gamma(y)}{z-y} \text { and } \quad \beta(y)=\limsup _{z \rightarrow y} \frac{\gamma(z)-\gamma(y)}{z-y} \quad \text { for } y \in \mathbb{R}
$$

and let $L_{y}$ and $U_{y}, 0<y<\infty$, be as in Remark 1.3 and Proposition 1.5, respectively. It is a simple matter to verify that if $0<y<\infty$ and $E \in \mathcal{M}(\Omega)$, then

$$
L_{y}(E)=\int_{E} \alpha(y-s(x)) d \mathcal{L}^{n} x \quad \text { and } \quad U_{y}(E)=\int_{E} \beta(y-s(x)) d \mathcal{L}^{n} x .
$$

In view of Theorem 1.3 the results of section 1.7 apply when $\alpha$ or $\beta$ and $s$ are sufficiently regular.

Of particular interest is when $1 \leq p<\infty$ and

$$
\gamma(y)=\frac{1}{p}|y|^{p} \quad \text { whenever } y \in \mathbb{R} .
$$

Rudin, Osher, and Fatemi [ROF] studied the case $p=2$ and Chan and Esedoğlu [CE] studied the case $p=1$. The results of section 1.7 will allow us to construct a number of interesting examples of minimizers in [AW2], a sequel to this paper; we believe these examples provide insights into the nature of total variation regularization. At the end of this paper we will determine $\mathbf{m}_{\epsilon}^{\text {loc }}(F)$ when $\Omega=\mathbb{R}^{2}, s$ is the indicator function of a square, and $\gamma$ is as in (1.10). Note that $F$ is strictly convex if $p>1$ and merely convex if $p=1$.

Suppose $K \in \mathbf{L}_{1}\left(\mathbb{R}^{n}\right)$. Let

$$
F(f)=\int_{\mathbb{R}^{n}} \gamma(K * f(x)-s(x)) d \mathcal{L}^{n} x \quad \text { for } f \in \mathcal{F}\left(\mathbb{R}^{n}\right) .
$$

It is easy to see that $F$ is admissible but not local except in degenerate situations. Nonetheless, the results of section 1.5 apply.

1.9. Some results on the Chan-Esedoglu functional. Suppose $s, \gamma, F$ are as in section 1.8 with $\gamma(y)=|y|$ for $y \in \mathbb{R}$. Whenever $0<y<\infty$ and $E \in \mathcal{M}(\Omega)$ we use (1.9) to obtain

$$
\begin{aligned}
& L_{y}(E)=\mathcal{L}^{n}(E \cap\{y>s\})-\mathcal{L}^{n}(E \cap\{y \leq s\})=\widehat{N_{\{y \leq s\}}}(E), \\
& U_{y}(E)=\mathcal{L}^{n}(E \cap\{y \geq s\})-\mathcal{L}^{n}(E \cap\{y<s\})=\widehat{N_{\{y<s\}}}(E),
\end{aligned}
$$

where for each $S \in \mathcal{M}(\Omega)$ we have set

$$
N_{S}(E)=\Sigma_{\Omega}(E, S) \quad \text { for } E \in \mathcal{M}(\Omega) .
$$

We use Theorem 1.4 to obtain interesting results about $N_{S}, S \in \mathcal{M}(\Omega)$, one of which is as follows; it was suggested by a similar result in a different context in [CA1] and will be used in [AW2] in determining $\mathbf{n}_{\epsilon}^{l o c}\left(N_{S}\right), 0<\epsilon<\infty$, for certain $S$, which 
in view of the above formulae for $L_{y}$ and $U_{y}, 0<y<\infty$, and Theorem 1.3 will allow us to determine $\mathbf{m}_{\epsilon}^{\text {loc }}(F), 0<\epsilon<\infty$, where $F(f)=\int_{\mathbb{R}^{2}}\left|f-1_{S}\right| d \mathcal{L}^{2}$ for $f \in \mathcal{F}\left(\mathbb{R}^{2}\right)$.

TheOREM 1.7. Suppose $S \in \mathcal{M}(\Omega), 0<\epsilon<\infty$, and $\mathcal{A}$ is a nonempty subfamily of $\mathbf{n}_{\epsilon}^{\text {loc }}\left(N_{S}\right)$. Then

$$
\cap \mathcal{A} \in \mathbf{n}_{\epsilon}^{\text {loc }}\left(N_{S}\right) \quad \text { and, provided } \mathcal{L}^{n}(\cup \mathcal{A})<\infty, \quad \cup \mathcal{A} \in \mathbf{n}_{\epsilon}^{\text {loc }}\left(N_{S}\right) .
$$

2. Geometric measure theoretic background. We find the mathematical infrastructure of normal and integral currents to be convenient in carrying out this work. For that reason we will adopt, for the most part, the notation and terminology of [FE]; note the extensive glossary, list of notation, and index starting on page 669 of that book. We avoided using that notation and terminology in the introduction in order to make it more accessible to readers not familiar with [FE].

2.1. More notations and conventions. Suppose $\mu$ measures $\Omega$, which is to say $\mu$ maps the power set of $\Omega$ countably subadditively into $[0, \infty]$; whenever $A \subset \Omega$ we let

$$
\mu\llcorner A(B)=\mu(A \cap B) \quad \text { whenever } B \subset \Omega
$$

and note that $\mu\llcorner A$ measures $\Omega$.

Whenever $f$ is a function mapping a subset of a normed vector space into another normed vector space, $a$ is an interior point of the domain of $f$, and $f$ is Fréchet differentiable at $a$, we let

$$
\partial f(a)
$$

be the Fréchet differential of $f$ at $a$.

If $V$ is a vector space, $v \in V$, and $\psi$ belongs to the dual space of $V$, we frequently write

$$
\langle v, \psi\rangle \text { instead of } \psi(v) .
$$

We use spt as an abbreviation for "support."

2.2. Spaces of smooth functions; currents. Whenever $Y$ is a Banach space we let

$$
\mathcal{E}(\Omega, Y) \text { and } \mathcal{D}(\Omega, Y)
$$

be the space of smooth $Y$ valued functions on $\Omega$ and the space of compactly supported members of $\mathcal{E}(\Omega, Y)$, respectively, with the strong topologies as described in [FE, sect. 4.1.1]. Thus $\mathcal{X}(\Omega)=\mathcal{D}\left(\Omega, \mathbb{R}^{n}\right)$.

We let

$$
\mathcal{E}(\Omega) \text { and } \mathcal{D}(\Omega)
$$

equal $\mathcal{E}(\Omega, \mathbf{R})$ and $\mathcal{D}(\Omega, \mathbf{R})$, respectively. For each nonnegative integer $m$ we let

$$
\mathcal{E}^{m}(\Omega) \text { and } \mathcal{D}^{m}(\Omega)
$$

equal $\mathcal{E}(\Omega, Y)$ and $\mathcal{D}(\Omega, Y)$, respectively, with $Y=\bigwedge^{m} \mathbb{R}^{n}$. Thus $\mathcal{E}^{m}(\Omega)$ is the space of smooth differential $m$-forms on $\Omega$, and $\mathcal{D}^{m}(\Omega)$ is the space of those members of $\mathcal{E}^{m}(\Omega)$ with compact support. We let

$$
\mathcal{E}_{m}(\Omega) \quad \text { and } \quad \mathcal{D}_{m}(\Omega)
$$


be the duals of $\mathcal{E}^{m}(\Omega)$ and $\mathcal{D}^{m}(\Omega)$, respectively. Thus $\mathcal{D}_{m}(\Omega)$ is the space of $m$ dimensional currents on $\Omega$, and $\mathcal{E}_{m}(\Omega)$ is the space of $m$-dimensional currents with compact support on $\Omega$. We define the boundary operator

$$
\partial: \mathcal{D}_{m}(\Omega) \rightarrow \mathcal{D}_{m-1}(\Omega)
$$

by setting $\partial T(\omega)=T(d \omega)$ whenever $T \in \mathcal{D}_{m}(\Omega)$ and $\omega \in \mathcal{D}_{m-1}(\Omega)$; here $d$ is exterior differentiation.

Suppose $T \in \mathcal{D}_{m}(\Omega)$. As in [FE, sect. 4.1.5] we let

$$
\|T\|,
$$

the total variation measure of $T$, be the largest Borel regular measure on $\Omega$ such that

$$
\|T\|(G)=\sup \left\{|T(\omega)|: \omega \in \mathcal{D}^{m}(\Omega),\|\omega\| \leq 1 \text { and } \operatorname{spt} \omega \subset G\right\}
$$

for each open subset $G$ of $\Omega$; here $\|\cdot\|$ is the comass which in case $m \in\{0,1, n-1, n\}$ is the Euclidean norm; these are the only cases we will encounter in this paper. It follows immediately from this definition that

$$
\|T\|(G) \leq \liminf _{\nu \rightarrow \infty}\left\|S_{\nu}\right\|(G) \text { for any open subset } G \text { of } \Omega
$$

whenever $S$ is a sequence in $\mathcal{D}_{m}(\Omega)$ such that $S_{\nu}(\omega) \rightarrow T(\omega)$ as $\nu \rightarrow \infty$ whenever $\omega \in \mathcal{D}^{m}(\Omega)$. We let

$$
\mathbf{M}(T)=\|T\|(\Omega)
$$

and call this nonnegative extended real number the mass of $T$. We say $T$ is representable by integration if $\|T\|$ is a Radon measure which is equivalent to the statement that $\|T\|(K)<\infty$ whenever $K$ is a compact subset of $\Omega$. If this is the case and $\vec{T}$ is the $\|T\|$ measurable function with values in $\left\{\xi \in \bigwedge_{m} \mathbb{R}^{n}:\|\xi\|=1\right\}$ defined in [FE, sect. 4.1.7], there is a unique extension of $T$ to the $\|T\|$ summable functions on $\Omega$ with values in $\bigwedge^{m} \mathbb{R}^{n}$, which we continue to denote by $T$, such that

$$
T(\omega)=\int\langle\vec{T}(x), \omega(x)\rangle d\|T\| x
$$

whenever $\omega$ is a $\|T\|$ summable function on $\Omega$ with values in $\bigwedge^{m} \mathbb{R}^{n}$. If $T \in \mathcal{D}_{m}(\Omega)$ is representable by integration, $l$ is a nonnegative integer not exceeding $m$, and $\eta$ is a bounded Borel function on $\Omega$ with values in $\bigwedge^{l} \mathbb{R}^{n}$, then we let

$$
T\left\llcorner\eta \in \mathcal{D}_{m-l}(\Omega)\right.
$$

be such that

$$
T\left\llcorner\eta(\omega)=\int\langle\vec{T}(x),(\eta \wedge \omega)(x)\rangle d\|T\| x \quad \text { for } \omega \in \mathcal{D}^{m-l}(\Omega) .\right.
$$

2.3. The current corresponding to a locally summable function. We let

$$
\mathbf{e}_{1}, \ldots, \mathbf{e}_{n} \text { and } \mathbf{e}^{1}, \ldots, \mathbf{e}^{n}
$$

be the standard basis vectors and covectors for $\mathbb{R}^{n}$ and its dual space, respectively. We let

$$
\mathbf{E}^{n}=\mathbf{e}^{1} \wedge \cdots \wedge \mathbf{e}^{n} \in \bigwedge^{n} \mathbb{R}^{n}
$$


be the standard orientation on $\mathbb{R}^{n}$.

We let

$$
\mathbf{V}^{n} \in \mathcal{D}^{n}(\Omega)
$$

be such that $\mathbf{V}^{n}(x)=\mathbf{E}^{n}$ for $x \in \Omega$.

Definition 2.1. Whenever $f \in \mathbf{L}_{1}^{\text {loc }}(\Omega)$ we define

$$
[f] \in \mathcal{D}_{n}(\Omega)
$$

by setting

$$
[f]\left(\phi \mathbf{V}^{n}\right)=\int_{\Omega} \phi f d \mathcal{L}^{n} \quad \text { whenever } \phi \in \mathcal{D}(\Omega) .
$$

Suppose $f \in \mathbf{L}_{1}^{\text {loc }}(\Omega)$. For any $X \in \mathcal{X}(\Omega)$ we have $\left.d(X\lrcorner \mathbf{V}^{n}\right)=(-1)^{n-1}(\operatorname{div} X) \mathbf{V}^{n}$ so that

$$
\left.\partial[f](X\lrcorner \mathbf{V}^{n}\right)=(-1)^{n-1} \int f \operatorname{div} X d \mathcal{L}^{n} ;
$$

here $\lrcorner$ is as in [FE, sect. 1.5.1]. It follows that

$$
\mathbf{T V}(f, B)=\|\partial[f]\|(B) \quad \text { whenever } B \text { is a Borel subset of } \Omega \text {. }
$$

2.4. Mapping currents. Whenever $T \in \mathcal{D}_{m}(\Omega)$ and $F$ is a smooth map from $\Omega$ to the open subset $\Gamma$ of some Euclidean space whose restriction to the support of $T$ is proper, we let

$$
F_{\#} T \in \mathcal{D}_{m}(\Gamma)
$$

be such that $F_{\#} T(\omega)=T\left(F^{\#} \omega\right)$ for any $\omega \in \mathcal{D}^{m}(\Gamma)$; here the pullback $F^{\#}$ is as in [FE, sect. 4.1.6]. If $F$ carries $\Omega$ diffeomorphically onto $\Gamma, T$ is representable by integration, and $\vec{T}(x)$ is decomposable for $\|T\|$ almost all $x \in \Omega$, then we have

$$
\int b(y) d\left\|F_{\#} T\right\| y=\int b(F(x))\left|\bigwedge_{m} \partial F(x)(\vec{T}(x))\right| d\|T\| x
$$

for nonnegative Borel function $b$ on $\Gamma$. By a simple approximation argument one need only assume that $F$ is of class $C^{1}$ if $T$ is representable by integration.

2.5. A mapping formula. Suppose $\Gamma$ is an open subset of $\mathbb{R}^{n} ; f \in \mathbf{L}_{1}^{\text {loc }}(\Omega)$; $F: \Omega \rightarrow \Gamma$ is locally Lipschitzian; the restriction of $F$ to the support of $[f]$ is proper; $A$ is the set of $y \in \Gamma$ such that $F^{-1}[\{y\}]$ is finite and such that if $F(x)=y$, then $F$ is differentiable at $x$; and $g: \Gamma \rightarrow \mathbb{R}$ is such that

$$
g(y)= \begin{cases}\sum_{x \in F^{-1}[\{y\}]} f(x) \operatorname{sgn} \operatorname{det} \partial F(x) & \text { if } y \in A, \\ 0 & \text { else. }\end{cases}
$$

Then $g \in \mathbf{L}_{1}^{\text {loc }}(\Gamma)$ and

$$
F_{\#}[f]=[g] .
$$

In particular, if $F$ is univalent and $\operatorname{det} \partial F(x)>0$ for $\mathcal{L}^{n}$ almost all $x \in \Omega$, then

$$
F_{\#}[f]=\left[f \circ F^{-1}\right] .
$$

See [FE, sect. 4.1.25] for the proof. 
2.6. Slicing. Suppose $m, l$ are positive integers, $m \geq l, T \in \mathcal{D}^{m}(\Omega), T$ is locally flat as defined in [FE, sect. 4.1.12], and $f: \Omega \rightarrow \mathbb{R}^{l}$ is locally Lipschitzian. Note that if both $T$ and $\partial T$ are representable by integration, then $T$ is locally flat; this will always be the case when we apply slicing in this paper. For $y \in \mathbb{R}^{l}$ we follow $[\mathrm{FE}$, sect. 4.3.1] and define

$$
\langle T, f, y\rangle
$$

the slice of $T$ in $f^{-1}[\{y\}]$ to be that member of $\mathcal{D}_{m-l}(\Omega)$ which, if it exists, satisfies

$$
\langle T, f, y\rangle(\psi)=\lim _{r \downarrow 0} \frac{T\left\llcorner\left[f^{\#}\left(\mathbf{B}^{l}(y, r) \wedge \mathbf{V}^{l}\right)\right](\psi)\right.}{\mathcal{L}^{l}\left(\mathbf{B}^{l}(y, r)\right)} \quad \text { whenever } \psi \in \mathcal{D}^{m-l}(\Omega),
$$

where $T\left\llcorner\left[f^{\#}\left(\mathbf{B}^{l}(y, r) \wedge \mathbf{V}^{l}\right)\right]\right.$ is defined as in [FE, sect. 4.3.1]. Then, by [FE, sect. 4.3.1], the slice $\langle T, f, y\rangle$ exists for $\mathcal{L}^{l}$ almost all $y$ and satisfies

$$
\operatorname{spt}\langle T, f, y\rangle \subset f^{-1}[\{y\}] \quad \text { and } \quad \partial\langle T, f, y\rangle=(-1)^{l}\langle\partial T, f, y\rangle .
$$

Moreover, we have from [FE, sect. 4.3.2] that

$$
\int \Phi(y)\langle T, f, y\rangle(\psi) d \mathcal{L}^{l} y=\left[T\left\llcorner f^{\#}\left(\Phi \wedge \mathbf{V}^{l}\right)\right](\psi)\right.
$$

whenever $\Phi$ is a bounded Borel function on $\mathbb{R}^{l}$ and $\psi \in \mathcal{D}^{m-l}(\Omega)$ and that

$$
\int\left(\int b\|\langle T, f, y\rangle\|\right) d \mathcal{L}^{l} y=\int b d\left\|T\left\llcorner f^{\#} \mathbf{V}^{l}\right]\right\|
$$

whenever $b$ is a nonnegative Borel function on $\Omega$.

Proposition 2.1. Suppose $K$ is a compact subset of $\Omega, u(x)=\operatorname{dist}(x, K)$ for $x \in \Omega, R$ is the supremum of the set of $r \in(0, \infty)$ such that $\{u \leq r\} \subset \Omega$, $f, g \in \mathbf{B V}^{l o c}(\Omega)$, and

$$
h_{r}=g 1_{\{u \leq r\}}+f 1_{\{u>r\}} \quad \text { for each } r \in(0, R) .
$$

Then $h_{r} \in \mathbf{B V}^{\text {loc }}(\Omega)$ for $\mathcal{L}^{1}$ almost all $r \in(0, R)$, and whenever $0<r<s<R$ we have

$$
\int_{r}^{s}\left\|\partial\left[h_{\rho}\right]\right\|(\{u \leq \rho\}) d \mathcal{L}^{1} \rho \leq \int_{\{r<u<s\}}|f-g| d \mathcal{L}^{n}+\int_{r}^{s}\|\partial[g]\|(\{u \leq \rho\}) d \mathcal{L}^{1} \rho .
$$

Proof. From [FE, sect. 4.2.1] and [FE, sect. 4.3.4] we find that

$$
\partial\left[h_{\rho}\right]=\langle[g]-[f], u, \rho\rangle+(\partial[g])\llcorner\{u \leq \rho\}+(\partial[f])\llcorner\{u>\rho\}
$$

for $\mathcal{L}^{1}$ almost all $\rho \in(0, R)$. Now multiply by $1_{\{u \leq \rho\}}$, integrate from $r$ to $s$, and invoke (2.9).

2.7. Densities and density ratios. Suppose $\mu$ measures $\Omega, m$ is a nonnegative integer, and $\alpha(m)=\mathcal{L}^{m}\left(\mathbf{U}^{m}(0,1)\right)$. For each $a \in \Omega$ we set

$$
\Theta^{m}(\mu, a, r)=\frac{\mu(\mathbf{B}(a, r))}{\alpha(m) r^{m}} \quad \text { whenever } 0<r<\operatorname{dist}\left(a, \mathbb{R}^{n} \sim \Omega\right)
$$

and

$$
\Theta^{m}(\mu, a)=\lim _{r \rightarrow 0} \Theta^{m}(\mu, a, r)
$$

provided this limit exists. 
2.8. Sets of finite perimeter. Suppose $E$ is a Lebesgue measurable subset of $\Omega$. Proceeding as in [FE, sect. 4.5.5], we say $u \in \mathbb{R}^{n}$ is an exterior normal to $E$ at $b \in \Omega$ if $|u|=1$ and

$$
\Theta^{n}\left(\mathcal{L}^{n}\llcorner\{x \in E:(x-b) \bullet u>0\} \cup\{x \in \Omega \sim E:(x-b) \bullet u<0\}, b)=0 .\right.
$$

We let

\section{$\mathbf{n}_{E}$}

be the set of $(b, u) \in \Omega \times \mathbb{R}^{n}$ such that either $u$ is an exterior normal to $E$ at $b$ or $u=0$ and there is no exterior normal to $E$ at $b$; note that $\mathbf{n}_{E}$ is a function with domain $\Omega$. We let

$$
\mathbf{b}(E)
$$

the reduced boundary of $E$, be equal to the set of points $b \in \Omega$ such that there is an exterior normal to $E$ at $b$.

TheOrem 2.1 (see [FE, sect. 4.5.6]). Suppose $E$ is a subset of $\Omega$ with locally finite perimeter. The following statements hold:

(i) $\mathbf{b}(E)$ is a Borel set which is countably $\left(\mathcal{H}^{n-1}, n-1\right)$ rectifiable.

(ii) $\|\partial[E]\|=\mathcal{H}^{n-1}\llcorner\mathbf{b}(E)$.

(iii) For $\mathcal{H}^{n-1}$ almost all $b \in \mathbf{b}(E)$ we have

$$
* \mathbf{n}_{E}(b)=\overrightarrow{\partial[E]}(b) \quad \text { and } \quad \Theta^{n-1}(\|\partial[E]\|, b)=1 ;
$$

here $*$ is the Hodge star operator as defined in [FE, sect. 1.7.8].

(iv) For $\mathcal{H}^{n-1}$ almost all $b \in \Omega \sim \mathbf{b}(E), \Theta^{n-1}(\|\partial[E]\|, b)=0$ and

$$
\text { either } \Theta^{n}\left(\mathcal { L } ^ { n } \llcorner E , b ) = 0 \quad \text { or } \quad \Theta ^ { n } \left(\mathcal{L}^{n}\llcorner(\Omega \sim E), b)=0\right.\right. \text {. }
$$

It follows that if $E$ is a subset of $\Omega$ with locally finite perimeter, then

$$
\left.\partial[E](X\lrcorner \mathbf{V}^{n}\right)=(-1)^{n-1} \int X \bullet \mathbf{n}_{E} d\|\partial[E]\| \quad \text { whenever } X \in \mathcal{X}(\Omega)
$$

Proposition 2.2. Suppose $E$ is a subset of $\Omega$ with finite perimeter and $C$ is a closed convex subset of $\mathbb{R}^{n}$. Then

$$
\mathbf{M}(\partial[C \cap E]) \leq \mathbf{M}(\partial[E]) .
$$

Proof. Let $\rho: \mathbb{R}^{n} \rightarrow C$ be such that $|x-\rho(x)|=\operatorname{dist}(x, C)$ for $x \in \mathbb{R}^{n}$. In case spt $[E]$ is compact we infer from $(2.6)$ that $[C \cap E]=\rho_{\#}[E]$ so that, as $\operatorname{Lip} \rho \leq 1$, (2.12) holds. In case spt $[E]$ is not compact we let $E_{r}=E \cap \mathbf{U}^{n}(0, r), 0<r<\infty$, and apply the result just obtained together with (2.10) and (2.1).

2.9. Basic facts about functions of bounded variation. Proofs of the following formulae, which are absolutely fundamental for this work, may be found in [FE, sect. 4.5.9, eq. (13)]. Suppose $f \in \mathbf{B V}^{l o c}(\Omega)$; then $\mathbb{R} \ni y \mapsto \partial[\{f \geq y\}](\omega)$ is $\mathcal{L}^{1}$ summable and

$$
\partial[f](\omega)=\int \partial[\{f>y\}](\omega) d \mathcal{L}^{1} y \quad \text { whenever } \omega \in \mathcal{D}^{n-1}(\Omega)
$$


moreover, if $B$ is a Borel subset of $\Omega$, then $\mathbb{R} \ni y \mapsto \| \partial\left[\{f>y\} \|(B)\right.$ is $\mathcal{L}^{1}$ measurable and

$$
\|\partial[f]\|(B)=\int\|\partial[\{f>y\}]\|(B) d \mathcal{L}^{1} y
$$

The following well-known theorem follows from (2.1) and the discussion in [FE, sect. 4.5.7] concerning locally flat currents of dimension $n$ in $\Omega$.

TheOREM 2.2 (compactness theorem). Suppose $C$ is a sequence of nonnegative real numbers and $K$ is a sequence of compact subsets of $\Omega$ such that $\cup_{\nu=1}^{\infty} K_{\nu}=\Omega$. Then

$$
\bigcap_{\nu=1}^{\infty}\left\{f \in \mathbf{B V}^{l o c}(\Omega): \int_{K_{\nu}}|f| d \mathcal{L}^{n}+\|\partial[f]\|\left(K_{\nu}\right) \leq C_{\nu}\right\}
$$

is a compact subset of $\mathbf{L}_{1}^{\text {loc }}(\Omega)$.

Proposition 2.3. Suppose $f \in \mathbf{B V}^{l o c}(\Omega)$ and $y \in \mathbf{R}$. Then $f \wedge y, f \vee y \in$ $\mathbf{B V}^{l o c}(\Omega)$ and

$$
\|\partial[f \wedge y]\|+\|\partial[f \vee y]\|=\|\partial[f]\| .
$$

Proof. Since $f+y=f \wedge y+f \vee y$ it is trivial that the right-hand side of (2.15) does not exceed the left-hand side of (2.15). Using (2.13) one readily shows that

$$
[f \wedge y](\omega)=\int_{-\infty}^{y}[\{f \geq z\}](\omega) d \mathcal{L}^{1} z \quad \text { and } \quad[f \vee y](\omega)=\int_{y}^{\infty}[\{f>y\}](\omega) d \mathcal{L}^{1} y
$$

whenever $\omega \in \mathcal{D}^{n}(\Omega)$. Applying $\partial$ one infers

$$
\|\partial[f \wedge y]\| \leq \int_{-\infty}^{y}\|\partial[\{f>y\}]\| d \mathcal{L}^{1} y \quad \text { and } \quad\|\partial[f \vee y]\| \leq \int_{y}^{\infty}\|\partial[\{f>y\}]\| d \mathcal{L}^{1} y .
$$

By (2.14) the sum of the right-hand sides of these inequalities is $\|\partial[f]\|$. Thus the left-hand side of (2.15) does not exceed the right-hand side.

2.10. The "layer cake" formula. Chan and Esedoglu in $[\mathrm{CE}]$ call the following elementary formula the "layer cake" formula; it is indispensable in this work.

Proposition 2.4. Suppose $f, g$ are real valued Lebesgue measurable functions on $\Omega$. Then

$$
\int_{\Omega}|f-g| d \mathcal{L}^{n}=\int_{-\infty}^{\infty} \Sigma_{\Omega}(\{f>y\},\{g>y\}) d \mathcal{L}^{1} y
$$

Proof. Apply Tonelli's theorem to calculate the $\mathcal{L}^{n} \times \mathcal{L}^{1}$ measure of $\{(x, y) \in$ $\Omega \times \mathbb{R}: g(x)<y \leq f(x)\}$ and $\{(x, y) \in \Omega \times \mathbb{R}: f(x)<y \leq g(x)\}$ and add the results.

3. Deformations and variations. We suppose throughout this section that

(i) $X: \Omega \rightarrow \mathbb{R}^{n}$ is continuously differentiable and $K=\operatorname{spt} X$ is compact;

(ii) $I$ is an open interval containing 0 such that if $t \in I$ and

$$
h_{t}(x)=x+t X(x) \quad \text { for } x \in \Omega,
$$

then $h_{t}$ carries $\Omega$ diffeomorphically (in the $C^{1}$ sense) onto itself; 
(iii) $D$ is a Lebesgue measurable subset of $\Omega$ with locally finite perimeter and

$$
E_{t}=\left\{h_{t}(x): x \in D\right\} \quad \text { for } t \in I
$$

(iv) for $x \in \mathbf{b}(D)$

$$
\begin{aligned}
& P(x) \text { is orthogonal projection of } \mathbb{R}^{n} \text { onto }\left\{v \in \mathbb{R}^{n}: v \bullet \mathbf{n}_{D}(x)=0\right\}, \\
& l_{1}(x)=P(x) \circ \partial X(x) \circ P(x) \text { and } \quad l_{2}(x)=P(x)^{\perp} \circ \partial X(x) \circ P(x) .
\end{aligned}
$$

Note that given $X$ as in (i) there is always $I$ as in (ii).

\subsection{Some useful variational formulae.}

Proposition 3.1. Suppose

$$
A(t)=\left\|\partial\left[E_{t}\right]\right\|(K) \quad \text { for each } t \in I .
$$

Then $A$ is smooth, and

$$
\dot{A}(0)=\int a_{1} d\|\partial[D]\| \quad \text { and } \quad \ddot{A}(0)=\int a_{2} d\|\partial[D]\|,
$$

where for $x \in \mathbf{b}(D)$ we have set

$$
a_{1}(x)=\operatorname{trace} l_{1}(x) \quad \text { and } \quad a_{2}(x)=a_{1}(x)^{2}+\operatorname{trace}\left(l_{2}(x)^{*} \circ l_{2}(x)-l_{1}(x) \circ l_{1}(x)\right) .
$$

Proof. It follows from (2.6) that $\left[E_{t}\right]=h_{t \#}[D]$ and therefore $\partial\left[E_{t}\right]=h_{t \#} \partial[D]$ for any $t \in I$. Now recall from Theorem 2.1(iii) that $* \mathbf{n}_{D}(x)=\overrightarrow{\partial[D]}(x)$ for $\|\partial[D]\|$ almost all $x$, differentiate under the integral sign in (2.5), and use the formulae

$$
\left.\left(\frac{d}{d t}\right)^{j} \bigwedge_{n-1} \partial h_{t}(x)(\overrightarrow{\partial[D]}(x))\right|_{t=0}=a_{j}(x), \quad j=1,2, \quad x \in \mathbf{b}(D),
$$

proofs of which may be found in [FE, sect. 5.1.8].

Since $\left[E_{t}\right]-[D]$ is compactly supported, $\left(\left[E_{t}\right]-[D]\right)\left(\phi \mathbf{V}^{n}\right)$ is well defined in the following proposition.

Proposition 3.2. For any $\phi \in \mathcal{E}(\Omega)$ we have

$$
\left(\left[E_{t}\right]-[D]\right)\left(\phi \mathbf{V}^{n}\right)=\int_{0}^{t}\left(\int \phi\left(h_{\tau}(x)\right) W_{\tau}(x) d\|\partial[D]\| x\right) d \mathcal{L}^{1} \tau,
$$

where, for each $t \in I$, we have set

$$
W_{t}(x)=\left\langle X(x) \wedge \bigwedge_{n-1} \partial h_{t}(x)\left(* \mathbf{n}_{D}(x)\right), \mathbf{E}^{n}\right\rangle \quad \text { for } x \in \mathbf{b}(D) .
$$

Proof. For each $t \in I$ let $J_{t}=[0, t] \in \mathcal{D}_{1}(\mathbb{R})$ as in [FE, sect. 4.1.8]. From [FE, sect. 4.1.8] we have $\left\|J_{t} \times \partial[D]\right\|=\left\|J_{t}\right\| \times\|\partial[D]\|$ for each $t \in I$. From [FE, sect. 4.1.8] and Theorem 2.1(iii) we have

$$
\overrightarrow{J_{t} \times \partial[D]}(\tau, x)=(1,0) \wedge \overrightarrow{\partial[D]}(x)=(1,0) \wedge * \mathbf{n}_{D}(x) \quad \text { for }(\tau, x) \in(0, t) \times \mathbf{b}(D) .
$$

Suppose $t \in I$. We obtain

$$
\left[E_{t}\right]-[D]=h_{t \#}[D]-[D]=h_{\#}\left(J_{t} \times \partial[D]\right)
$$


from the homotopy formula of [FE, sect. 4.1.9]; the formula to be proved now follows from (2.5).

Proposition 3.3. Suppose $\mathcal{L}^{n}(D)<\infty, \zeta \in \mathbf{L}_{\infty}(\Omega)$, and

$$
B(t)=\int_{E_{t}} \zeta d \mathcal{L}^{n} \quad \text { for } t \in I .
$$

If $\zeta$ is continuous, then $B$ is continuously differentiable and

$$
\dot{B}(0)=\int \zeta\left(X \bullet \mathbf{n}_{D}\right) d\|\partial[D]\| .
$$

If $\zeta$ is continuously differentiable, then $B$ is twice continuously differentiable and

$$
\ddot{B}(0)=\int(\zeta Y+(\nabla \zeta \bullet X) X) \bullet \mathbf{n}_{D} d\|\partial[D]\|,
$$

where for $x \in \mathbf{b}(D)$ we have set

$$
Y(x)=\left(\operatorname{trace} l_{1}(x)\right) X(x)-l_{2}(x)(X(x)) .
$$

Proof. Using straightforward approximations if necessary, we may assume that $\zeta$ is smooth. For each $t \in I$ and $x \in \mathbf{b}(D)$ let

$$
\xi_{t}(x)=\bigwedge_{n-1} \partial h_{t}(x)\left(* \mathbf{n}_{D}(x)\right) \quad \text { and } \quad W_{t}(x)=\left\langle\dot{h}_{t}(x) \wedge \xi_{t}(x), \mathbf{E}^{n}\right\rangle .
$$

Suppose $x \in \mathbf{b}(D)$. Let $u_{1}, \ldots, u_{n}$ be an orthonormal sequence of vectors in $\mathbb{R}^{n}$ such that $u_{1}=\mathbf{n}_{D}(x)$ and $* u_{1}=u_{2} \wedge \cdots \wedge u_{n}$; since $\left\langle u_{1} \wedge * u_{1}, \mathbf{E}^{n}\right\rangle=1$ we have

$$
\left\langle w \wedge u_{2} \wedge \cdots \wedge u_{n}, \mathbf{E}^{n}\right\rangle=w \bullet u_{1}\left\langle u_{1} \wedge * u_{1}, \mathbf{E}^{n}\right\rangle=w \bullet u_{1} \quad \text { for any } w \in \mathbb{R}^{n} ;
$$

see [FE, sect. 1.7.8] for the properties of $*$.

It should now be clear from Proposition 3.2 that (3.1) holds.

Let $u^{1}, \ldots, u^{n}$ be the sequence of covectors dual to $u_{1}, \ldots, u_{n}$ and let $\omega_{1}, \ldots, \omega_{n}$ be those covectors such that $\partial X(x)=\sum_{j=1}^{n} \omega_{j} u_{j}$. We have

$$
\begin{aligned}
\left.\frac{d}{d t} W_{t}(x)\right|_{t=0}= & \left.X(x) \wedge \frac{d}{d t} \xi_{t}(x)\right|_{t=0} \\
= & \left.X(x) \wedge \sum_{i=2}^{n} \partial X(x)\left(u_{i}\right) \wedge\left(u^{i}\right\lrcorner \xi_{0}(x)\right) \\
= & \left.X(x) \wedge \sum_{i=2}^{n} \sum_{j=1}^{n}\left\langle u_{i}, \omega_{j}\right\rangle u_{j} \wedge\left(u^{i}\right\lrcorner \xi_{0}(x)\right) \\
= & \left.X(x) \wedge \sum_{i=1}^{n}\left\langle u_{i}, \omega_{i}\right\rangle u_{i} \wedge\left(u^{i}\right\lrcorner \xi_{0}(x)\right) \\
& \left.\quad+X(x) \wedge \sum_{i=2}^{n}\left\langle u_{i}, \omega_{1}\right\rangle u_{1} \wedge\left(u^{i}\right\lrcorner \xi_{0}(x)\right) \\
= & \left(\left(\operatorname{trace} l_{1}(x) X(x)-l_{2}(x)(X(x))\right) \bullet \mathbf{n}_{D}(x)\right) u_{1} \wedge * u_{1},
\end{aligned}
$$

so (3.2) holds. 
4. Second fundamental forms and mean curvature. Suppose $M$ is an embedded hypersurface of class $C^{2}$ in $\Omega$.

The second fundamental form of $M$ is the function $\Pi$ on $M$ whose value at $a \in M$ is a linear map from $\operatorname{Nor}(M, a)$ into the symmetric linear maps from $\operatorname{Tan}(M, a)$ to itself characterized by the requirement that if $U$ is an open subset of $\mathbb{R}^{n}, a \in U \cap M$; $N: U \rightarrow \mathbb{R}^{n} ; N$ is of class $C^{1} ;$ and $N(x) \in \operatorname{Nor}(M, x)$ whenever $x \in U \cap M$, then

$$
\Pi(a)(N(a))(v) \bullet w=\partial N(a)(v) \bullet w \quad \text { for } v, w \in \operatorname{Tan}(M, a) .
$$

The mean curvature vector of $M$ is, by definition, the function $H$ on $M$ whose value at a point $a$ of $M$ is that member $H(a)$ of $\operatorname{Nor}(M, a)$ whose inner product with $u \in \operatorname{Nor}(M, a)$ is the trace of $\Pi(a)(u)$. In the classical literature the mean curvature vector is $1 /(n-1)$ times $H$ as defined here, hence the word "mean." It turns out the factor $1 /(n-1)$ is inconvenient when one is working, as we will be, with the first variation of area; for this reason we omit it. The direction of the mean curvature vector, and not just its magnitude, will be important in this work.

If $a \in M$, the length of $\Pi(a)$ is, by definition, the square root of the sum of the squares of the eigenvalues of $\Pi(a)(u)$ whenever $u \in \operatorname{Nor}(M, a)$ and $|u|=1$.

Suppose $f: \Omega \rightarrow \mathbb{R}$ is $C^{2} ; \nabla f(x) \neq 0$ whenever $x \in \Omega ; y$ is in the range of $f$; and $M=\{f=y\}$, so $M$ is an embedded hypersurface of class $C^{2}$ in $\Omega$. It follows that if $a \in M$ then

$$
\Pi(a)(\nabla f(a))(u) \bullet v=\partial(\nabla f)(a)(u) \bullet v \quad \text { whenever } u, v \in \operatorname{Tan}(M, a) .
$$

Suppose $\Omega=\mathbb{R}^{n} \sim\{0\}, f(x)=|x|^{2} / 2$ for $x \in \Omega, 0<R<\infty$, and $M=\{x \in$ $\left.\mathbb{R}^{n}:|x|=R\right\}$. Then $\nabla f(x)=x$ for $x \in \Omega$. It follows that if $a \in M$ then

$$
\Pi(a)(a)(v) \bullet w=\frac{v \bullet w}{|a|} \quad \text { whenever } v, w \in \operatorname{Tan}(M, a), \quad H(a)=\frac{n-1}{R^{2}} a
$$

and the length $\Pi(a)$ equals the square root of $(n-1) / R^{2}$.

5. The spaces $\mathcal{B}_{\lambda}(\Omega)$ and $\mathcal{C}_{\lambda}(\Omega), 0 \leq \lambda<\infty$. We suppose throughout this section that $0 \leq \lambda<\infty$, and we study the spaces $\mathcal{B}_{\lambda}(\Omega)$ and $\mathcal{C}_{\lambda}(\Omega)$.

5.1. Basic results on $\mathcal{B}_{\lambda}(\Omega)$ and $\mathcal{C}_{\lambda}(\Omega)$. In what follows we will frequently make use of the following simple observation. Suppose $f, g \in \mathbf{L}_{1}^{\text {loc }}(\Omega), K$ is a compact subset of $\Omega, g \in \mathbf{k}(f, K)$, and $y \in \mathbb{R}$. Then

$$
\{g>y\} \in \mathbf{k}(\{f>y\}) .
$$

Moreover, $\geq$ may be replaced by any of $\leq,>$, and $<$.

Remark 5.1. It is an elementary corollary of Theorem 5.1 below that if $D$ is an open subset of $\Omega$ with smooth boundary $M$ and $D \in \mathcal{C}_{\lambda}(\Omega)$, then the length of the mean curvature vector of $M$ does not exceed $\lambda$. The converse of this statement is false as one sees in case $\lambda=0$ by considering a set whose boundary is an unstable minimal surface.

However, if $f: \Omega \rightarrow \mathbb{R}$ is smooth with nowhere vanishing gradient and, for each $y$ in the range of $f$, the length of the mean curvature vector of $\{f=y\}$ never exceeds $\lambda$, then a simple calibration argument shows that $f \in \mathcal{B}_{\lambda}(\Omega)$.

Lemma 5.1. Suppose $f \in \mathcal{B}_{\lambda}(\Omega), g \in \mathbf{B V}^{\text {loc }}(\Omega), K$ is a compact subset of $\Omega$, $u(x)=\operatorname{dist}(x, K)$ for $x \in \Omega, 0<h<\infty$, and $\{u \leq h\}$ is a compact subset of $\Omega$. 
Then

$$
\|\partial[f]\|(K) \leq\|\partial[g]\|(\{u \leq h\})+\left(\lambda+\frac{1}{h}\right) \int_{\{u \leq h\}}|f-g| d \mathcal{L}^{n} .
$$

In particular,

$$
\|\partial[f]\|(K) \leq\left(\lambda+\frac{1}{h}\right) \int_{\{u \leq h\}}|f-y| d \mathcal{L}^{n} \quad \text { for } y \in \mathbb{R} .
$$

Proof. For each $r \in(0, h)$ let $h_{r}=g 1_{\{u \leq r\}}+f 1_{\{u>r\}}$. Then $h_{r} \in \mathbf{k}(f,\{u \leq r\})$ and $f-h_{r}=(f-g) 1_{\{u \leq r\}}$, so

$$
\|\partial[f]\|(\{u \leq r\}) \leq\left\|\partial\left[h_{r}\right]\right\|(\{u \leq r\})+\lambda \int_{\{u \leq r\}}|f-g| .
$$

Now integrate this inequality from 0 to $h$ and make use of (2.10) to prove the first inequality; to obtain the second, set $g(x)=y$ for $x \in \Omega$.

TheOrem 5.1. Suppose $\lambda \in[0, \infty), f \in \mathcal{B}_{\lambda}(\Omega)$, and $y \in \mathbf{R}$. Then

$$
\{f+y, y f, f \wedge y, f \vee y\} \subset \mathcal{B}_{\lambda}(\Omega) .
$$

Proof. Suppose $K$ is a compact subset of $\Omega$. Obviously, $0 f=0 \in \mathcal{B}_{\lambda}(\Omega)$. Suppose $y \in \mathbb{R} \sim\{0\}$ and $g \in \mathbf{k}(y f, K)$. Then $g / y \in \mathbf{k}(f, K)$, so

$$
\begin{aligned}
\|\partial[y f]\|(K) & =|y|\|\partial[f]\|(K) \\
& \leq|y|\left(\|\partial[g / y]\|(K)+\lambda \int_{\Omega}|f-g / y| d \mathcal{L}^{n}\right) \\
& =\|\partial[g]\|(K)+\lambda \int_{\Omega}|y f-g| d \mathcal{L}^{n} .
\end{aligned}
$$

Thus $y f \in \mathcal{B}_{\lambda}(\Omega)$.

Suppose $g \in \mathbf{k}(f+y, K)$. Then $g-y \in \mathbf{k}(f, K)$, so

$$
\begin{aligned}
\|\partial[f+y]\|(K) & =\|\partial[f]\|(K) \\
& \leq\|\partial[g-y]\|(K)+\lambda \int_{\Omega}|f-(g-y)| d \mathcal{L}^{n} \\
& =\|\partial[g]\|(K)+\lambda \int_{\Omega}|(f+y)-g| d \mathcal{L}^{n}
\end{aligned}
$$

and thus $f+y \in \mathcal{B}_{\lambda}(\Omega)$.

Suppose $g \in \mathbf{k}(f \wedge y, K)$. Let $h=g+(f \vee y)-y$. Then $f-h=f+y-(f \vee y)-g=$ $f \wedge y-g$, so $h \in \mathbf{k}(f, K)$. Using Proposition 2.3 we estimate

$$
\begin{aligned}
\|\partial[f \wedge y]\|(K) & +\|\partial[f \vee y]\|(K) \\
& =\|\partial[f]\|(K) \\
& \leq\|\partial[h]\|(K)+\lambda \int_{K}|f-h| d \mathcal{L}^{n} \\
& \leq\|\partial[g]\|(K)+\|\partial[f \vee y]\|(K)+\lambda \int_{K}|f \wedge y-g| d \mathcal{L}^{n}
\end{aligned}
$$


and conclude that $f \wedge y \in \mathcal{B}_{\lambda}(\Omega)$.

Finally, $f \vee y=-((-f) \wedge(-y)) \in \mathcal{B}_{\lambda}(\Omega)$.

TheOREM 5.2. Suppose $\lambda \in[0, \infty), f$ is a sequence in $\mathcal{B}_{\lambda}(\Omega), F \in \mathbf{L}_{1}^{\text {loc }}(\Omega)$, and $f_{\nu} \rightarrow F$ in $\mathbf{L}_{1}^{\text {loc }}(\Omega)$. Then $F \in \mathcal{B}_{\lambda}(\Omega)$ and

$$
\left\|\partial\left[f_{\nu}\right]\right\| \rightarrow\|\partial[F]\| \quad \text { weakly as } \nu \rightarrow \infty .
$$

Proof. Let $K$ be a compact subset of $\Omega$, let $u(x)=\operatorname{dist}(x, K)$ for $x \in \Omega$, and let $R=\sup \{r \in(0, \infty):\{u \leq r\} \subset \Omega\}$.

Suppose $h \in(0, R)$ and for each positive integer $\nu$ let $y_{\nu}$ be the average of $f_{\nu}$ on $\{u \leq h\}$. Let $Y$ be the average value of $F$ on $\{u \leq h\}$. From Lemma 5.1 we obtain

$$
\left\|\partial\left[f_{\nu}\right]\right\|(K) \leq\left(\lambda+\frac{1}{h}\right) \int_{\{u \leq h\}}\left|f_{\nu}-y_{\nu}\right| d \mathcal{L}^{n} \rightarrow\left(\lambda+\frac{1}{h}\right) \int_{\{u \leq h\}}|F-Y| d \mathcal{L}^{n}
$$

as $\nu \rightarrow \infty$. Since $K$ is arbitrary we infer from $(2.1)$ that $F \in \mathbf{B V}^{l o c}(\Omega)$.

For any $r \in(0, R)$ we infer from Lemma 5.1 that

$$
\left\|\partial\left[f_{\nu}\right]\right\|(K) \leq\|\partial[F]\|(\{u \leq r\})+\left(\lambda+\frac{1}{h}\right) \int_{\{u \leq h\}}\left|f_{\nu}-F\right| d \mathcal{L}^{n}
$$

for any positive integer $\nu$. Keeping in mind (2.1) we conclude that $\left\|\partial\left[f_{\nu}\right]\right\|$ converges weakly to $\|\partial[F]\|$ as $\nu \rightarrow \infty$.

We now show that $F \in \mathcal{B}_{\lambda}(\Omega)$. To this end, let $G \in \mathbf{B V}^{\text {loc }}(\Omega) \cap \mathbf{k}(F, K)$. For each positive integer $\nu$ and each $\rho \in(0, R)$ we let

$$
g_{\nu, \rho}=G\left\llcorner\{u \leq \rho\}+f_{\nu}\llcorner\{u>\rho\},\right.
$$

we note that $g_{\nu, \rho} \in \mathbf{k}\left(f_{\nu},\{u \leq \rho\}\right)$ and $f_{\nu}-g_{\nu, \rho}=\left(f_{\nu}-G\right) 1_{\{u \leq \rho\}}$, and we conclude that

$$
\left\|\partial\left[f_{\nu}\right]\right\|(\{u \leq \rho\}) \leq \| \partial\left[g_{\nu, \rho} \|(\{u \leq \rho\})+\lambda \int_{\{u \leq \rho\}}\left|G-f_{\nu}\right| d \mathcal{L}^{n} .\right.
$$

Suppose $0<r<R$ and $\nu$ is a positive integer. Keeping in mind that $G-f_{\nu}=F-f_{\nu}$ at $\mathcal{L}^{n}$ almost all points of $\Omega \sim K$, we integrate this inequality from 0 to $r$ and use (2.10) to obtain

$$
\begin{aligned}
r\left\|\partial\left[f_{\nu}\right]\right\|(K) \leq & \int_{0}^{r}\left\|\partial\left[f_{\nu}\right]\right\|(\{u \leq \rho\}) d \mathcal{L}^{1} \rho \\
\leq & \int_{\{0<u<r\}}\left|F-f_{\nu}\right| d \mathcal{L}^{n}+r\|\partial[G]\|(\{u \leq r\}) \\
& \quad+\lambda r \int_{\{u \leq r\}}\left|G-f_{\nu}\right| d \mathcal{L}^{n} .
\end{aligned}
$$

Letting $\nu \rightarrow \infty$ we find that

$$
\limsup _{\nu \rightarrow \infty}\left\|\partial\left[f_{\nu}\right]\right\|(K) \leq\|\partial[G]\|(\{u \leq r\})+\lambda \int_{\{u \leq r\}}|G-F| d \mathcal{L}^{n} .
$$

Letting $r \downarrow 0$ we infer that

$$
\|\partial[F]\|(K) \leq\|\partial[G]\|(K)+\lambda \int_{K}|G-F| d \mathcal{L}^{n},
$$

as desired. 
THEOREM 5.3. The following statements hold:

(i) If $f \in \mathcal{B}_{\lambda}(\Omega)$ and $y \in \mathbb{R}$, then $\{f>y\} \in \mathcal{C}_{\lambda}(\Omega)$.

(ii) If $f \in \mathbf{B V}^{\text {loc }}(\Omega), D=\left\{y \in \mathbb{R}:\{f>y\} \in \mathcal{C}_{\lambda}(\Omega)\right\}$, and $D$ is dense in $\mathbb{R}$, then $f \in \mathcal{B}_{\lambda}(\Omega)$.

(iii) If $\mathcal{E}$ is a nonempty nested subfamily of $\mathcal{C}_{\lambda}(\Omega)$, then $\cup \mathcal{E}$ and $\cap \mathcal{E}$ belong to $\mathcal{C}_{\lambda}(\Omega)$.

(iv) $E \in \mathcal{C}_{\lambda}(\Omega)$ if and only if $1_{E} \in \mathcal{B}_{\lambda}(\Omega)$ whenever $E \subset \Omega$.

Proof. We begin with a lemma.

Lemma 5.2. Suppose $f \in \mathbf{B V}^{l o c}(\Omega), D=\left\{y \in \mathbb{R}:\{f>y\} \in \mathcal{C}_{\lambda}(\Omega)\right\}$, and $\mathcal{L}^{1}(\mathbb{R} \sim D)=0$. Then $f \in \mathcal{B}_{\lambda}(\Omega)$.

Proof. Suppose $K$ is a compact subset of $\Omega$ and $g \in \mathbf{B V}^{\text {loc }}(\Omega) \cap \mathbf{k}(f, K)$. Keeping in mind (5.1) we infer from (2.14) and (2.16) that

$$
\begin{aligned}
\|\partial[f]\|(K) & =\int_{-\infty}^{\infty}\left\|\partial\left[1_{\{f>y\}}\right]\right\|(K) d \mathcal{L}^{1} y \\
& \leq \int_{-\infty}^{\infty}\left(\left\|\partial\left[1_{\{g>y\}}\right]\right\|(K)+\lambda \int\left|1_{\{f>y\}}-1_{\{g>y\}}\right| d \mathcal{L}^{n}\right) d \mathcal{L}^{1} y \\
& =\|\partial[g]\|(K)+\lambda \int|f-g| d \mathcal{L}^{n} .
\end{aligned}
$$

Suppose $E \in \mathcal{C}_{\lambda}(\Omega)$. Evidently, $\left\{1_{E}>y\right\} \in \mathcal{C}_{\lambda}(\Omega)$ for all $y \in \mathbb{R}$ so, by the lemma, $1_{E} \in \mathcal{B}_{\lambda}(\Omega)$. It being trivial that $\left\{E: 1_{E} \in \mathcal{B}_{\lambda}(\Omega)\right\}$ is a subset of $\mathcal{C}_{\lambda}(\Omega)$, we find that (iv) holds.

Suppose $\mathcal{E}$ is a nonempty nested subfamily of $\mathcal{C}_{\lambda}(\Omega)$. Choose a nondecreasing sequence $A$ and a nonincreasing sequence $B$ in $\mathcal{E}$ such that $1_{A_{\nu}} \rightarrow 1_{\cup \mathcal{E}}$ and $1_{B_{\nu}} \rightarrow 1_{\cap \mathcal{E}}$ in $\mathbf{L}_{1}^{\text {loc }}(\Omega)$, as $\nu \rightarrow \infty$. From Theorem 5.2 we infer that the indicator functions of $\cup \mathcal{E}$ and $\cap \mathcal{E}$ belong to $\mathcal{B}_{\lambda}(\Omega)$, so (iii) now follows from (iv).

Suppose $f$ and $D$ are as in (ii). Since $D$ is dense in $\mathbb{R}$ we have for any $y \in \mathbb{R}$ that

$$
\{f>y\}=\cup_{z \in(y, \infty) \cap D}\{f>z\},
$$

so $\{f>y\} \in \mathcal{C}_{\lambda}(\Omega)$ by (iii). The lemma now implies (ii).

Finally, suppose $f \in \mathcal{B}_{\lambda}(\Omega)$ and $y \in \mathbb{R}$. For each positive integer $\nu$ let

$$
g_{\nu}=\nu\left(\left((f-y) \wedge \frac{1}{\nu}\right) \vee 0\right)
$$

and note that $g_{\nu} \in \mathcal{B}_{\lambda}(\Omega)$ by Theorem 5.1. One readily verifies that $g_{\nu} \uparrow 1_{\{f>y\}}$ as $\nu \uparrow \infty$ so that, by Theorem $5.2,1_{\{f>y\}} \in \mathcal{B}_{\lambda}(\Omega)$, so $\{f>y\} \in \mathcal{C}_{\lambda}(\Omega)$ by (iv), and thus (i) holds.

\subsection{Generalized mean curvature.}

Proposition 5.1. Suppose $\lambda \in[0, \infty), D \in \mathcal{C}_{\lambda}(\Omega)$, and $X \in \mathcal{X}(\Omega)$. Then

$$
\int \operatorname{trace} P(x) \circ \partial X(x) \circ P(x) d\|\partial[D]\| x \leq \lambda \int|X| d\|\partial[D]\|,
$$

where, for each $x \in \mathbf{b}(D)$, we have let $P(x)$ be an orthogonal projection of $\mathbb{R}^{n}$ onto $\left\{v \in \mathbb{R}^{n}: v \bullet \mathbf{n}_{D}(x)=0\right\}$.

Remark 5.2. We restate this theorem in the language of [AW1]. Let $V$ be the $(n-1)$-dimensional varifold in $\Omega$ naturally associated to $\partial[D]$ as in [AW1, sect. 3.5]; the preceding theorem says that

$$
\|\delta V\| \leq \lambda\|V\|
$$


where $\delta V$ is as in [AW1, sect. 4.2].

Proof. Let us adopt the notation of section 3. In particular, $A(t)=\left\|\partial\left[E_{t}\right]\right\|(K)$ for $t \in I$. For any positive $t \in I$ we infer from Proposition 3.2 that

$$
\frac{A(t)-A(0)}{t} \leq \frac{\lambda}{t}\left\|\left[E_{t}\right]-[D]\right\|(K) \leq \frac{1}{t} \lambda \int_{0}^{t}\left(\int|X|\left\|\partial \dot{h}_{\tau}(x)\right\|^{n-1} d\|\partial[D]\| x\right) d \mathcal{L}^{1} \tau
$$

The estimate to be proved now follows from Proposition 3.1.

\subsection{Consequences of the monotonicity theorem.}

Theorem 5.4. Suppose $\lambda \in[0, \infty), D \in \mathcal{C}_{\lambda}(\Omega), a \in \Omega$, and $R=\operatorname{dist}\left(a, \mathbb{R}^{n} \sim\right.$ $\Omega)$. Then

(i) $(0, R) \ni r \mapsto e^{\lambda r} \Theta^{n-1}(\|\partial[D]\|, a, r)$ is nondecreasing;

(ii) $\Theta^{n-1}(\|\partial[D]\|, a)$ exists and depends uppersemicontinuously on a;

(iii) $\Theta^{n-1}(\|\partial[D]\|, a) \geq 1$ if $a \in \operatorname{spt} \partial[D]$;

if $a \in \mathbf{s p t}[D]$, we have

(iv) $e^{-\lambda r} \alpha(n-1) r^{n-1} \leq\|\partial[D]\|\left(\mathbf{U}^{n}(a, r)\right)$ whenever $0<r<R$;

(v) $e^{-\lambda r} \frac{\alpha(n-1)}{n} r^{n} \leq(1+\lambda r) \mathcal{L}^{n}\left(D \cap \mathbf{U}^{n}(a, r)\right)$ whenever $0<r<R$.

Proof. In view of Remark 5.2, (i) follows from the monotonicity theorem of [AW1, sect. 5.1]. (i) clearly implies (ii). (iii) is a consequence of Theorem 2.1(ii) and (iii). (iv) follows directly from (i) and (iii).

Suppose $0<r<R$. For each $\rho \in(0, r)$ let $E_{\rho}=D \cap\{u>\rho\}$, where we have set $u(x)=|x-a|$ for $x \in \Omega$ and note that $E_{\rho} \in \mathbf{k}(E,\{u \leq \rho\})$, so

$$
\begin{aligned}
e^{-\lambda r} \alpha(n-1) \rho^{n-1} & \leq e^{-\lambda \rho} \alpha(n-1) \rho^{n-1} \\
& \leq\|\partial[D]\|(\{u \leq \rho\}) \\
& \leq\left\|\partial\left[E_{\rho}\right]\right\|(\{u \leq \rho\})+\lambda \Sigma_{\Omega}\left(E_{\rho}, D\right) .
\end{aligned}
$$

Now integrate this inequality over $(0, r)$ and make use of $(2.10)$, with $f$ and $g$ there equal to $1_{E}$ and 0 , respectively.

Remark 5.3. It follows from (iv) that if $\Omega=\mathbb{R}^{n}$ and $\mathcal{L}^{n}(D)<\infty$, then spt $[D]$ is compact.

Corollary 5.1. Suppose $0<R<\infty, 0<r<\infty, a \in \Omega, R+r \leq \operatorname{dist}\left(a, \mathbb{R}^{n} \sim\right.$ $\Omega), f \in \mathcal{B}_{\lambda}(\Omega)$, and

$$
Y=\left\{y \in \mathbb{R}:\|\partial[\{f>y\}]\|\left(\mathbf{U}^{n}(a, R)\right)>0\right\} .
$$

Then

$$
\mathcal{L}^{1}(Y) e^{-\lambda r} \alpha(n-1) r^{n-1} \leq\|\partial[f]\|\left(\mathbf{U}^{n}(a, R+r)\right)
$$

and

$$
\mathcal{L}^{1}(Y) e^{-\lambda r} \frac{\alpha(n-1)}{n} r^{n} \leq(1+\lambda r) \int_{\mathbf{U}^{n}(a, R+r)}|f| d \mathcal{L}^{n}
$$

Proof. For each $y \in Y \sim\{0\}$ we apply Theorem 5.4 with $D$ there equal to $\{f>y\}$ to a ball of radius $r$ with center at a point $b$, where $\Theta^{n-1}(\|\partial[\{f>y\}]\|, b)=1$. 
5.4. Proof of the regularity theorem for $\mathcal{C}_{\lambda}(\Omega)$. In view of the regularity theorem of $[\mathrm{AW} 1$, sect. 8] the present regularity theorem, Theorem 1.2, will follow from the following lemma.

Lemma 5.3. Suppose

$$
1<\zeta<\infty .
$$

There exists $\eta \in(0,1)$ such that if $0 \leq \lambda<\infty, a \in \mathbb{R}^{n}, 0<R<\infty$,

$$
\lambda R \leq \eta, \quad E \in \mathcal{C}_{\lambda}\left(\mathbf{U}^{n}(a, R)\right), \quad \text { and } \quad a \in \operatorname{spt} \partial[E],
$$

then

$$
\Theta^{n-1}(\|\partial[E]\|, a, \eta R) \leq \zeta .
$$

Proof. Due to the way the various entities in the lemma change under application of homotheties and translations, we find that we may assume without loss of generality that $a=0$ and $R=1$.

Suppose the lemma were false. Then there would exist $\zeta \in(1, \infty)$; a sequence $\eta$ in $(0,1)$ with limit zero; and sequences $E, \lambda$ such that, for each positive integer $\nu$,

$$
\lambda_{\nu} \leq \eta_{\nu}, \quad E_{\nu} \in \mathcal{C}_{\lambda_{\nu}}\left(\mathbf{U}^{n}(0,1)\right), \quad \text { and } \quad 0 \in \mathbf{s p t} \partial\left[E_{\nu}\right]
$$

but such that

$$
\Theta^{n-1}\left(\left\|\partial\left[E_{\nu}\right]\right\|, 0, \eta_{\nu}\right)>\zeta
$$

From the monotonicity theorem we have

$$
(0,1) \ni t \mapsto e^{\lambda_{\nu} t} \Theta^{n-1}\left(\left\|\partial\left[E_{\nu}\right]\right\|, 0, t\right) \quad \text { is nondecreasing }
$$

for each positive integer $\nu$.

Replacing $E$ by a subsequence if necessary we may use Theorems 2.2 and 5.2 to obtain a Lebesgue measurable subset $F$ of $\mathbf{U}^{n}(0,1)$ such that $E_{\nu} \rightarrow F$ in $\mathbf{L}_{1}^{\text {loc }}\left(\mathbf{U}^{n}(0,1)\right)$ as $\nu \rightarrow \infty$,

$$
F \in \bigcap_{\nu=1}^{\infty} \mathcal{C}_{\lambda_{\nu}}\left(\mathbf{U}^{n}(0,1)\right)=\mathcal{C}_{0}\left(\mathbf{U}^{n}(0,1)\right)
$$

and

$$
\left\|\partial\left[E_{\nu}\right]\right\| \rightarrow\|\partial[F]\| \quad \text { weakly as } \nu \rightarrow \infty .
$$

Letting $B$ equal the set of $t \in(0,1)$ such that $\|\partial[F]\|\left(\left\{x \in \mathbb{R}^{n}:|x|=t\right\}\right)$ is positive we observed that $B$ is countable and infer from (5.5) and (2.1) that

$$
\lim _{\nu \rightarrow \infty} \Theta^{n-1}\left(\| \partial\left[E_{\nu}\right], 0, t\right)=\Theta^{n-1}(\| \partial[F], 0, t) \quad \text { for any } t \in(0,1) \sim B .
$$

This together with (5.2), Theorem 5.4, and the fact that $\lambda_{\nu} \rightarrow 0$ as $\nu \rightarrow \infty$ implies

$$
\Theta^{n-1}(\|\partial[F]\|, 0, t) \geq \zeta \quad \text { whenever } t \in(0,1) \sim B .
$$

As $F \in \mathcal{C}_{0}\left(\mathbf{U}^{n}(0,1)\right)$ we find that $\partial[F]$ is an absolutely area minimizing integral current of dimension $n-1$ in $\mathbf{U}^{n}(0,1)$. As Theorem 2.1 implies that

$$
\Theta^{n-1}(\|\partial[F]\|, x)=1 \quad \text { for }\|\partial[F]\| \text { almost all } x
$$

it follows from the regularity theorem of [FE, sect. 5.4.15] that $\partial[F]$ is integration over an oriented $(n-1)$-dimensional real analytic hypersurface $M$ of $\mathbf{U}^{n}(0,1)$. Consequently, $\Theta^{n-1}(\|\partial[F]\|, 0)=1$, which is incompatible with (5.6). 
5.4.1. The case $\boldsymbol{n}=2$. One can do a little better than the preceding theorem if $n=2$, as follows. Let $\mathbf{w}(m)=\sqrt{1+m^{2}}$ for $m \in \mathbf{R}$. Suppose $V$ and $W$ are nonempty open intervals, $g: V \rightarrow W$ is continuously differentiable, $0 \leq \lambda<\infty$, and

$$
D=\{(v, w) \in V \times W: w<g(v)\} \in \mathcal{C}_{\lambda}(V \times W) .
$$

We will show that

$$
\operatorname{Lip}\left(\mathbf{w}^{\prime} \circ g^{\prime}\right) \leq \lambda .
$$

Note that if $g$ is twice differentiable, then $\left(\mathbf{w}^{\prime} \circ g^{\prime}\right)^{\prime}$ is the curvature function of the graph of $g$.

We prove (5.7) as follows. Suppose $\phi \in \mathcal{D}(V)$ and for each $t \in \mathbb{R}$ let $g_{t}=g+t \phi$. Let $I$ be an open interval containing 0 such that if $t \in I$, then $g_{t}(v) \in W$ whenever $v \in V$. Let $E_{t}=\left\{(v, w) \in V \times W: w<g_{t}(v)\right\}$ for $t \in I$. We have

$$
\left(\|\partial[D]\|-\left\|\partial\left[E_{t}\right]\right\|\right)(V \times W) \leq \lambda \Sigma_{V \times W}\left(D, E_{t}\right) .
$$

Now

$\lim _{t \downarrow 0} \frac{1}{t}\left(\|\partial[D]\|-\left\|\partial\left[E_{t}\right]\right\|\right)(V \times W)=-\left.\int_{I} \frac{d}{d t} \mathbf{w}\left(g^{\prime}+t \phi^{\prime}\right)\right|_{t=0} d \mathcal{L}^{1}=-\int_{I}\left(\mathbf{w}^{\prime} \circ g^{\prime}\right) \phi^{\prime} d \mathcal{L}^{1}$

and

$$
\lim _{t \downarrow 0} \frac{1}{t} \Sigma_{V \times W}\left(D, E_{t}\right)=\int_{V}|\phi| d \mathcal{L}^{1}
$$

To obtain (5.7) we let $\phi$ approximate plus or minus one times the indicator function of a compact subinterval of $V$.

6. Locality. Suppose $M$ and $\mu$ are as in Proposition 1.3.

6.1. Proof of Proposition 1.3. If $M$ has a representation as in (1.2) where $m$ is a bounded Borel function, it is trivial that $M$ is local.

Suppose $M$ is local. Then $\|\mu\|_{\mathbf{L}_{\infty}(\Omega)} \leq \mathbf{l}(M)$, which implies there is a unique Radon measure on $\Omega$ whose restriction to $\mathcal{M}(\Omega)$ equals $\hat{M}$. That (1.2) holds with $m=\mu$ follows from the theory of symmetrical derivation; see, for example, [FE, sect. 2.9].

6.2. Proof of Proposition 1.4. If $F$ has a representation as in (1.3) where $k$ satisfies (i) and (ii) of Proposition 1.4, it is trivial that $M$ is local, and it follows from the theory of symmetrical derivation that for $0<y<\infty$ we have $k(x, y)=\kappa(x, y)$ for $\mathcal{L}^{n}$ almost all $x \in \Omega$.

Suppose $F$ is local. For any $y \in(0, \infty)$ we have that $\mathcal{M}(\Omega) \ni E \mapsto \hat{F}\left(y 1_{E}\right)$ is local so that, by Proposition 1.3,

$$
\hat{F}\left(y 1_{E}\right)=\int_{E} \kappa(x, y) d \mathcal{L}^{n} x \quad \text { for } E \in \mathcal{M}(\Omega) .
$$

Given $f \in \mathcal{F}(\Omega)$ and $0=y_{0}<y_{1}<y_{2}<\cdots<y_{N}<\infty$ we infer from the locality of $F$ that

$$
\hat{F}\left(\sum_{i=1}^{N} y_{i} 1_{\left\{y_{i-1}<f \leq y_{i}\right\}}\right)=\sum_{i=1}^{N} \hat{F}\left(y_{i} 1_{\left\{y_{i-1}<f \leq y_{i}\right\}}\right)=\sum_{i=1}^{N} \int_{\left\{y_{i-1}<f \leq y_{i}\right\}} \kappa\left(x, y_{i}\right) d \mathcal{L}^{n} x,
$$

from which the representation for $F(f)$ in (1.3) easily follows using the admissibility of $F$, and (i) and (ii) of Proposition 1.4 hold with $k=\kappa$. 
6.3. Proof of Proposition 1.5. (i), (ii), and (iii) are immediate.

For any $x \in \Omega$ we have that $(0, \infty) \ni y \mapsto \kappa(x, y)$ is absolutely continuous so that

$$
\kappa(x, f(x))=\int_{0}^{f(x)} u(x, z) d \mathcal{L}^{1} z=\int_{0}^{\infty} u(x, z) 1_{\{f \geq z\}}(x) d \mathcal{L}^{1} z .
$$

Integrating this equation over $\Omega$ and invoking Fubini's theorem, we infer that

$$
\int_{\Omega} \kappa(x, f(x)) d \mathcal{L}^{n} x=\int_{0}^{\infty}\left(\int_{\{f \geq z\}} u(x, z) d \mathcal{L}^{n} x\right) d \mathcal{L}^{1} z .
$$

For each $E \in \mathcal{M}(\Omega)$ let $\zeta(E)$ be the set of $y \in(0, \infty)$ such that

$$
U_{y}(E)=\int_{E} u(x, y) d \mathcal{L}^{n} x .
$$

Since $(0, \infty) \ni y \mapsto \hat{F}\left(y 1_{E}\right)$ is absolutely continuous we find that

$$
\hat{F}\left(y 1_{E}\right)=\int_{0}^{y} U_{y}(E) d \mathcal{L}^{1} y \quad \text { whenever } y \in(0, \infty) .
$$

Now assume $F$ is local. Applying Proposition 1.4 together with (6.1) with $f$ equal to $y 1_{E}$, we find that

$$
\int_{0}^{y}\left(\int_{E} u(x, z) d \mathcal{L}^{n} x\right) d \mathcal{L}^{1} z=\int_{0}^{y} U_{z}(E) d \mathcal{L}^{1} z \quad \text { for } y \in(0, \infty),
$$

which implies that $\mathcal{L}^{1}((0, \infty) \sim \zeta(E))=0$. Let $\mathcal{E}$ be a countable subfamily of $\mathcal{M}(\Omega)$ which is dense with respect to $\Sigma_{\Omega}(\cdot, \cdot)$ and let $Z=\cap\{\zeta(E): E \in \mathcal{E}\}$. Since $\mathcal{M}(\Omega) \ni$ $E \mapsto U_{y}(E)$ and $\mathcal{M}(\Omega) \ni E \mapsto \int_{E} u(x, y) d \mathcal{L}^{n} x$ are Lipschitzian with respect to $\Sigma_{\Omega}(\cdot, \cdot)$, we find that

$$
U_{y}(E)=\int_{E} u(x, y) d \mathcal{L}^{n} x \quad \text { whenever } y \in Z \text { and } E \in \mathcal{M}(\Omega) .
$$

Since $\mathcal{L}^{1}((0, \infty) \sim Z)=0$ we find that (iv) of Proposition 1.5 holds.

Suppose $f \in \mathcal{F}(\Omega)$. Use (1.3) with $k=\kappa$ to represent $F(f)$. (v) now follows from (6.1) and (6.2).

6.4. Proof of Proposition 1.6. That (i) implies (ii) is immediate. That (ii) implies (iii) is a direct consequence of the subadditivity of limsup. That (iii) implies (i) follows directly from (v) of Proposition 1.5. Thus (i), (ii), and (iii) are equivalent.

We leave the proof of the following elementary lemma to the reader.

Lemma 6.1. Suppose $g: \mathbb{R} \rightarrow \mathbb{R}, g$ is absolutely continuous, and

$$
h(y)=\liminf _{z \rightarrow y} \frac{g(z)-g(y)}{z-y} \quad \text { for } y \in \mathbb{R} .
$$

Then $g$ is convex if and only if $h$ is nondecreasing. Moreover, if $g$ is convex, then $h$ is right continuous.

The lemma implies that (iii) and (v) are equivalent. Since the admissibility of $F$ implies that $\mathbb{R} \ni y \mapsto \hat{F}\left(y_{E}\right)$ is locally Lipschitzian for any $E \in \mathcal{M}(\Omega)$, the lemma implies that (ii) and (iv) are equivalent.

The final assertion follows from the right continuity assertion of the lemma. 
6.5. The class $\mathcal{G}(\Omega)$. Let

$$
p: \Omega \times(0, \infty) \rightarrow \Omega \quad \text { and } \quad q: \Omega \times(0, \infty) \rightarrow(0, \infty)
$$

carry $(x, y) \in \Omega \times(0, \infty)$ to $x$ and $y$, respectively.

Whenever $G$ is an $\mathcal{L}^{n} \times \mathcal{L}^{1}$ measurable subset of $\Omega \times(0, \infty)$ we let

$$
[G] \in \mathcal{D}_{n+1}(\Omega \times(0, \infty))
$$

be as in (2.2), with $\mathbf{V}^{n}$ there replaced by $\left(p^{\#} \mathbf{V}^{n}\right) \wedge d q$; that is,

$$
[G]\left(\psi\left(p^{\#} \mathbf{V}^{n}\right) \wedge d q\right)=\int_{G} \psi d\left(\mathcal{L}^{n} \times \mathcal{L}^{1}\right) \quad \text { whenever } \psi \in \mathcal{D}(\Omega \times(0, \infty)) .
$$

DeFinition 6.1. We let

$$
\mathcal{G}(\Omega)
$$

be the family of Lebesgue measurable subsets $G$ of $\Omega \times(0, \infty)$ such that

$$
\left(\mathcal{L}^{n} \times \mathcal{L}^{1}\right)(G)<\infty \quad \text { and } \quad q[\operatorname{spt}[G]] \text { is bounded } .
$$

Note that if $G \in \mathcal{G}(\Omega)$, then for $\mathcal{L}^{1}$ almost all $y \in(0, \infty)$ we have $\{x:(x, y) \in$ $G\} \in \mathcal{M}(\Omega)$.

Definition 6.2. Whenever $G \in \mathcal{G}(\Omega)$ we let

$$
G^{\downarrow}: \Omega \rightarrow \mathbb{R}
$$

be such that

$$
G^{\downarrow}(x)= \begin{cases}\mathcal{L}^{1}(\{y:(x, y) \in G\}) & \text { if }\{y:(x, y) \in G\} \in \mathcal{M}((0, \infty)), \\ 0 & \text { otherwise. }\end{cases}
$$

Note that $G^{\downarrow} \in \mathcal{F}(\Omega)$ and $\int_{\Omega} G^{\downarrow} d \mathcal{L}^{n}=\left(\mathcal{L}^{n} \times \mathcal{L}^{1}\right)(G)$.

Definition 6.3. Whenever $f: \Omega \rightarrow[0, \infty)$ we let

$$
f^{\uparrow}=\{(x, y) \in \Omega \times(0, \infty): f(x)>y\} .
$$

Suppose $f: \Omega \rightarrow[0, \infty)$. Evidently,

$$
f \in \mathcal{F}(\Omega) \Leftrightarrow f^{\uparrow} \in \mathcal{G}(\Omega) .
$$

Tonelli's theorem implies that

$$
\left[\left(f^{\uparrow}\right)^{\downarrow}\right]=[f] \quad \text { whenever } f \in \mathcal{F}(\Omega) .
$$

Proposition 6.1. Suppose $G \in \mathcal{G}(\Omega), \phi \in \mathcal{D}(\Omega)$, and $\Psi \in \mathcal{E}((0, \infty))$. Then

$$
\begin{aligned}
p_{\#} & \left(\partial[G]\llcorner\Psi \circ q)\left(\phi \mathbf{V}^{\mathbf{n}}\right)\right. \\
& =(-1)^{n}[G]\left(p^{\#}\left(\phi \mathbf{V}^{n}\right) \wedge\left(\Psi^{\prime} \circ q\right) d q\right) \\
& =(-1)^{n} \int_{\Omega} \phi(x)\left(\int_{\{y:(x, y) \in G\}} \Psi^{\prime} d \mathcal{L}^{1}\right) d \mathcal{L}^{n} x .
\end{aligned}
$$


Proof. The first equation follows from the fact that

$$
d\left((\Psi \circ q) \wedge p^{\#}\left(\phi \mathbf{V}^{n}\right)\right)=\left(\Psi^{\prime} \circ q\right) d q \wedge p^{\#}\left(\phi \mathbf{V}^{n}\right),
$$

and the second follows from Fubini's theorem.

Corollary 6.1. Suppose $G \in \mathcal{G}(\Omega)$. Then

$$
\left[G^{\downarrow}\right]=(-1)^{n} p_{\#}\left((\partial[G])\llcorner q) \quad \text { and } \quad \partial\left[G^{\downarrow}\right]=(-1)^{n+1} p_{\#}((\partial[G])\llcorner d q) .\right.
$$

Proof. Letting $\Psi(y)=y$ for $y \in \mathbb{R}$ in the preceding proposition we deduce the first equation; the second equation is an immediate consequence of the first.

Proposition 6.2. Suppose $G \in \mathcal{G}(\Omega)$ and $\partial[G]$ is representable by integration. Then

$$
\left\|\partial\left[G^{\downarrow}\right]\right\|(B) \leq \int_{0}^{\infty}\|\partial[\{x:(x, y) \in G\}]\|(B) d \mathcal{L}^{1} y \quad \text { for any Borel subset } B \text { of } \Omega .
$$

Proof. Suppose $U$ is an open subset of $\Omega, \omega \in \mathcal{D}^{n-1}(\Omega), \operatorname{spt} \omega \subset U$, and $|\omega| \leq 1$. For each $y \in(0, \infty)$ let $i_{y}(x)=(x, y)$ for $x \in \Omega$. From [FE, sect. 4.3.8] we have

$$
\langle[G], q, y\rangle=i_{y_{\#}}[\{x:(x, y) \in G\}] \text { for } \mathcal{L}^{1} \text { almost all } y .
$$

From Corollary 6.1, (2.8), and (2.7) we find that

$$
\begin{aligned}
(-1)^{n+1} \partial\left[G^{\downarrow}\right](\omega) \mid & =\left((\partial[G])\llcorner d q)\left(p^{\#} \omega\right)\right. \\
& =\int_{0}^{\infty}\langle\partial[G], q, y\rangle\left(p^{\#} \omega\right) d \mathcal{L}^{1} y \\
& =-\int_{0}^{\infty} \partial[\{x:(x, y) \in G\}](\omega) d \mathcal{L}^{1} y \\
& \leq \int_{0}^{\infty}\|\partial[\{x:(x, y) \in G\}]\|(U) d \mathcal{L}^{1} y
\end{aligned}
$$

from which the inequality to be proved immediately follows.

6.6. Proof of Theorems 1.3 and 1.4. We now assume $F: \mathcal{F}(\Omega) \rightarrow \mathbb{R}, F$ is local, and $F$ is convex. In order to prove the fundamental theorems, Theorems 1.3 and 1.4, we will use $F$ to define a functional $F^{\uparrow}$ on subsets of $\Omega \times \mathbb{R}$, which will be very useful in analyzing $\mathbf{n}_{\epsilon}^{\text {loc }}(F)$. This is one of the main new ideas of the paper.

We leave to the reader the elementary proof of the following proposition.

Proposition 6.3. Suppose $G \in \mathcal{G}(\Omega)$. Then

$$
(0, \infty) \ni y \mapsto U_{y}(\{x:(x, y) \in G\}) \text { is } \mathcal{L}^{1} \text { summable. }
$$

DeFinition 6.4. Let

$$
F^{\uparrow}: \mathcal{G}(\Omega) \rightarrow \mathbb{R}
$$

be such that

$$
F^{\uparrow}(G)=F(0)+\int_{0}^{\infty} U_{y}(\{x:(x, y) \in G\}) d \mathcal{L}^{1} y \quad \text { whenever } G \in \mathcal{G}(\Omega) .
$$

We have a useful comparison principle. 
Theorem 6.1. We have

$$
F\left(G^{\downarrow}\right) \leq F^{\uparrow}(G) \quad \text { whenever } G \in \mathcal{G}(\Omega) .
$$

Proof. As we shall see, the theorem will follow rather directly from the following lemma.

Lemma 6.2. Suppose $a \in \Omega$ and $E \in \mathcal{M}((0, \infty))$. Then

$$
\kappa\left(a, \mathcal{L}^{1}(E)\right) \leq \int_{E} u(a, y) d \mathcal{L}^{1} y .
$$

Proof. Suppose $\phi \in \mathcal{D}((0, \infty))$ and $0 \leq \phi \leq 1$. Let $\Phi \in \mathcal{E}((0, \infty))$ be such that $\Phi^{\prime}=\phi$ and $\lim _{y \downarrow 0} \Phi(y)=0$. Then

$$
0 \leq \Phi(y) \leq y \quad \text { if } 0<y<\infty .
$$

Thus, as $(0, \infty) \ni y \mapsto \kappa(a, y)$ is absolutely continuous and $(0, \infty) \ni y \mapsto u(a, y)$ is nondecreasing, we have

$$
\kappa(a, \Phi(y))=\int_{0}^{y} u(a, \Phi(y)) \phi(y) d \mathcal{L}^{1} y \leq \int_{0}^{y} u(a, y) \phi(y) d \mathcal{L}^{1} y \quad \text { for } 0<y<\infty .
$$

We complete the proof by letting $\phi$ approximate the indicator function of $E$.

From the lemma we infer that

$$
\kappa\left(x, G^{\downarrow}(x)\right) \leq \int_{\{y:(x, y) \in G\}} u(x, y) d \mathcal{L}^{1} y \quad \text { for } \mathcal{L}^{n} \text { almost all } x \in \Omega .
$$

Integrating this inequality over $\Omega$ we use (iv) and (v) of Proposition 1.5 to obtain

$$
F\left(G^{\downarrow}\right)-F(0) \leq \int_{0}^{\infty} U_{y}(\{x:(x, y) \in G\}) d \mathcal{L}^{1} y=F^{\uparrow}(G)-F(0),
$$

as desired.

6.7. Proof of Theorem 1.3. We may assume without loss of generality that $F=\hat{F}$. For each $y \in(0, \infty)$ we let $D_{y}=\{f>y\}$.

Suppose $0<b<\infty, K$ is a compact subset of $\Omega$, and $E \in \mathbf{k}\left(D_{b}, K\right)$. We need to show that

$$
\epsilon\left\|\partial\left[D_{b}\right]\right\|(K)+U_{b}\left(D_{b}\right) \leq \epsilon\|\partial[E]\|(K)+U_{b}(E) .
$$

Let $u(x)=\operatorname{dist}(x, K)$ for $x \in \Omega$ and let $R$ be the supremum of the set of $r \in(0, \infty)$ such that $\{v \leq r\} \subset \Omega$. For each $(y, r) \in(0, \infty) \times(0, R)$ let

$$
\begin{aligned}
C_{y, r} & =(E \cap\{v \leq r\}) \cup\left(D_{y} \cap\{v>r\}\right) \in \mathcal{M}(\Omega), \\
a(y, r) & =\epsilon\left\|\partial\left[D_{y}\right]\right\|(\{v \leq r\})+U_{y}\left(D_{y}\right), \\
b(y, r) & =\epsilon\left\|\partial\left[C_{r, y}\right]\right\|(\{v \leq r\})+U_{y}\left(C_{r, y}\right) .
\end{aligned}
$$

Let

$$
W=\{(y, r) \in(0, \infty) \times(0, R): a(y, r) \leq b(y, r)\} .
$$

Lemma 6.3. For $\mathcal{L}^{1}$ almost all $y \in(0, \infty)$ we have

$$
a(y, r) \leq b(y, r)\} \quad \text { for } \mathcal{L}^{1} \text { almost all } r \in(0, R) .
$$


Proof. Suppose $r \in(0, R), B$ is a Borel subset of $(0, \infty)$, and

$$
G=\left\{(x, y) \in \Omega \times((0, \infty) \sim B): x \in D_{y}\right\} \cup\left\{(x, y) \in \Omega \times B: x \in C_{y, r}\right\} .
$$

Evidently, $G^{\downarrow}(x)=f(x)$ for $\mathcal{L}^{n}$ almost all $x \in\{v>r\}$, from which it follows that

$$
\epsilon\|\partial[f]\|(\{v \leq r\})+F(f) \leq \epsilon\left\|\partial\left[G^{\downarrow}\right]\right\|(\{v \leq r\})+F\left(G^{\downarrow}\right) .
$$

Let

$$
P=\int_{(0, \infty) \sim B}\left\|\partial\left[D_{y}\right]\right\|(\{v \leq r\}) d \mathcal{L}^{1} y \quad \text { and } \quad Q=\int_{(0, \infty) \sim B} U_{y}\left(D_{y}\right) d \mathcal{L}^{1} y .
$$

We have

$\|\partial[f]\|(\{v \leq r\})=P+\int_{B} \| \partial\left[D_{y} \|(\{v \leq r\}) d \mathcal{L}^{1} y \quad\right.$ and $\quad F(f)=Q+\int_{B} U_{y}\left(D_{y}\right) d \mathcal{L}^{1} y$.

From Corollary 6.1 and Proposition 6.2 we obtain

$$
\begin{aligned}
\left\|\partial\left[G^{\downarrow}\right]\right\|(\{v \leq r\}) & \leq \| \partial[G]\llcorner d q \|(\{v \leq r\} \times(0, \infty)) \\
& =\int\|\partial[\{x:(x, y) \in G\}]\|(\{v \leq r\}) d \mathcal{L}^{1} y \\
& =P+\int_{B}\left\|\partial\left[C_{y, r}\right]\right\|(\{v \leq r\}) .
\end{aligned}
$$

From (6.1) we obtain

$$
F\left(G^{\downarrow}\right) \leq F^{\uparrow}(G)=Q+\int_{B} U_{y}\left(C_{r, y}\right) d \mathcal{L}^{1} y
$$

which implies

$$
\int_{B} a(y, r) d \mathcal{L}^{1} y \leq \int_{B} b(y, r) d \mathcal{L}^{1} y .
$$

Owing to the arbitrariness of $B$ we infer that $a(y, r) \leq b(y, r)$ for $\mathcal{L}^{1}$ almost all $y \in(0, \infty)$, so the lemma follows from Tonelli's theorem.

We have $\left(D_{y} \sim D_{b}\right) \cup\left(D_{b} \sim D_{y}\right)=\{b<f \leq y\}$ whenever $b<y<\infty$, so that

$$
\lim _{y \downarrow b} \Sigma_{\Omega}\left(D_{y}, D_{b}\right)=0 .
$$

This implies that

$$
\lim _{y \downarrow b} \Sigma_{\Omega \sim K}\left(D_{y}, E\right)=\lim _{y \downarrow b} \Sigma_{\Omega \sim K}\left(D_{y}, D_{b}\right)=0 .
$$

By Proposition 1.5(iv) we have

$$
\begin{aligned}
\left|U_{y}\left(D_{y}\right)-U_{b}\left(D_{b}\right)\right| & \leq\left|U_{y}\left(D_{y}\right)-U_{y}\left(D_{b}\right)\right|+\left|U_{b}\left(D_{y}\right)-U_{b}\left(D_{b}\right)\right| \\
& \leq \mathbf{l}(F, Y) \Sigma_{\Omega}\left(D_{y}, D_{b}\right)+\left|U_{b}\left(D_{y}\right)-U_{b}\left(D_{b}\right)\right|
\end{aligned}
$$

as well as

$$
\left|U_{y}\left(C_{y, r}\right)-U_{y}(E)\right| \leq \mathbf{l}(F, Y) \Sigma_{\{u>r\}}\left(D_{y}, E\right) \leq \mathbf{l}(F, Y) \Sigma_{\Omega \sim K}\left(D_{y}, D_{b}\right)
$$


whenever $0<y<Y<\infty$. With the help of (6.6) and Proposition 1.6 we infer that

$$
\lim _{y \downarrow b} U_{y}\left(D_{y}\right)=U_{b}\left(D_{b}\right) .
$$

Suppose $0<r<R$. Since (6.6) and (2.1) imply that

$$
\left\|\partial\left[D_{b}\right]\right\|(K) \leq \liminf _{y \downarrow b}\left\|\partial\left[D_{y}\right]\right\|(\{u \leq \rho\}) \quad \text { for } 0<\rho<R,
$$

we infer from (6.9) that

$$
r\left(\left\|\partial\left[D_{b}\right]\right\|(K)+U_{b}\left(D_{b}\right)\right) \leq \liminf _{y \downarrow b} \int_{0}^{r} a(y, \rho) d \mathcal{L}^{1} \rho .
$$

Applying (2.10), with $f$ there equal to $1_{D_{y}}$ and $g$ there equal to $1_{E}$, and using (6.6), (6.7), (6.8), and (6.9), we find that

$$
\begin{aligned}
\int_{0}^{r} b(y, \rho) d \mathcal{L}^{1} \rho & \leq \Sigma_{\{u>r\}}\left(D_{y}, E\right)+\int_{0}^{r}\|\partial[E]\|(\{u \leq \rho\}) d \mathcal{L}^{1} \rho+\int_{0}^{r} U_{y}\left(C_{y, \rho}\right) d \mathcal{L}^{1} \rho \\
& \rightarrow \int_{0}^{r}\|\partial[E]\|(\{u \leq \rho\})+U_{b}(E) d \mathcal{L}^{1} \rho \quad \text { as } y \downarrow b .
\end{aligned}
$$

Using Lemma 6.2 and Tonelli's theorem we may choose a sequence $y$ in $(b, \infty)$ with limit $b$ such that

$$
\mathcal{L}^{1}\left(\left\{r \in(0, R):\left(y_{\nu}, r\right) \notin W\right\}\right)=0 \quad \text { for } \nu=1,2,3, \ldots .
$$

Thus

$$
\int_{0}^{r} a\left(y_{\nu}, \rho\right) d \mathcal{L}^{1} \rho \leq \int_{0}^{r} b\left(y_{\nu}, \rho\right) d \mathcal{L}^{1} \rho
$$

so (6.10) and (6.11) imply

$$
r\left(\left\|\partial\left[D_{b}\right]\right\|(K)+U_{b}\left(D_{b}\right)\right) \leq \int_{0}^{r}\|\partial[E]\|(\{u \leq \rho\})+U_{b}(E) d \mathcal{L}^{1} \rho ;
$$

dividing by $r$ and letting $r \downarrow 0$ we obtain (6.5).

We leave it to the reader to modify the proof just given in a straightforward way to show that $\{f \geq b\} \in \mathbf{n}_{\epsilon}^{\text {loc }}\left(L_{b}\right)$.

6.8. Proof of Theorem 1.4. Let $K$ be a compact subset of $\Omega$ and let $g \in \mathcal{F}(\Omega)$ such that spt $\left[G^{\downarrow}-g\right] \subset K$.

Suppose $y \in(0, \infty)$. Since $G^{\downarrow}(x)=g(x)$ for $\mathcal{L}^{n}$ almost all $x \in \Omega \sim K$ we find that

$$
\operatorname{spt}\left[\left\{G^{\downarrow}>y\right\}\right]-[\{g>y\}] \subset K,
$$

so that if $\{x:(x, y) \in G\} \in \mathbf{n}_{\epsilon}^{l o c}\left(U_{y}\right)$, we have

$$
\left\|\partial\left[\left\{G^{\downarrow}>y\right\}\right]\right\|(K)+U_{y}\left(\left\{G^{\downarrow}>y\right\}\right) \leq\|\partial[\{g>y\}]\|(K)+U_{y}(\{g>y\}) .
$$


Integrating over $y \in(0, \infty)$ with respect to $\mathcal{L}^{1}$ and using Proposition 6.2, Theorem 6.1, (2.6), and Proposition 1.5(v), we find that

$$
\begin{aligned}
\left\|\partial\left[G^{\downarrow}\right]\right\| & (K)+F\left(G^{\downarrow}\right) \\
& \leq \int_{0}^{\infty}\left\|\partial\left[\left\{G^{\downarrow}>y\right\}\right]\right\|(K) d \mathcal{L}^{1} y+F^{\uparrow}(G) \\
& =\int_{0}^{\infty}\left\|\partial\left[\left\{G^{\downarrow}>y\right\}\right]\right\|(K)+U_{y}\left(\left\{G^{\downarrow}>y\right\}\right) d \mathcal{L}^{1} y \\
& \leq \int_{0}^{\infty}\|\partial[\{g>y\}]\|(K)+U_{y}(\{g>y\}) d \mathcal{L}^{1} y \\
& =\|\partial[g]\|(K)+F(g) .
\end{aligned}
$$

It remains to deal with (1.6). For each $E \in \mathcal{M}(\Omega)$ let $C(E)$ be the set of $y \in(0, \infty)$ such that $L_{y}(E) \neq U_{y}(E)$. Since $(0, \infty) \ni \mapsto F\left(y 1_{E}\right)$ is convex we find that $C(E)$ is countable. Now choose a countable subfamily $\mathcal{E}$ of $\mathcal{M}(\Omega)$ which is dense with respect to the pseudometric $\Sigma_{\Omega}(\cdot, \cdot)$. By a straightforward approximation argument, which we leave to the reader, we find that $L_{y}(D)=U_{y}(D)$ whenever $D \in \mathcal{M}(\Omega)$ and $y \notin \cup\{C(E): E \in \mathcal{E}\}$.

7. Proof of Theorem 1.6. Theorem 1.6 will be proved by calculating the appropriate first and second variations, invoking the regularity theorem for $\mathcal{C}_{\lambda}(\Omega)$, and then utilizing higher regularity results for the minimal surface equation.

For each $x \in \mathbf{b}(D)$ we let $P(x)$ equal the orthogonal projection of $\mathbb{R}^{n}$ onto $\{v \in$ $\left.\mathbb{R}^{n}: v \bullet \mathbf{n}_{D}(x)=0\right\}$.

We may assume without loss of generality that $U=\Omega$. It follows from Proposition 1.2 and Theorem 1.2 that $\Sigma_{\Omega}(D, \Gamma)=0$, so $[D]=[\Gamma]$.

Part 1. Suppose $a \in M$. From Proposition 1.2 and Theorem 1.2 there are $\Psi, V, r, g$ such that $\Psi$ carries $\mathbb{R}^{n-1} \times \mathbb{R}$ isometrically onto $\mathbb{R}^{n}, \Psi(0,0)=a, V$ is an open subset of $\mathbb{R}^{n-1}, 0 \in V, 0<r<\infty, g: V \rightarrow(-r, r)$ is of class $C^{1, \mu}, \Psi[V \times(-r, r)] \subset \Omega$, and

$$
\Gamma \cap \Psi[V \times(-r, r)]=\Psi[\{(v, w) \in V \times(-r, r): w<g(v)\}] .
$$

Suppose $\phi \in \mathcal{D}(V)$. Choose an open interval $I$ such that $0 \in I$ and $g(v)+t \phi(v) \in$ $(-r, r)$ whenever $(v, t) \in V \times I$. For each $t \in I$ let

$$
\Phi(t)=\epsilon \int_{V} \sqrt{1+|\nabla(g+t \phi)|^{2}} d \mathcal{L}^{n-1}+\int_{V}\left(\int_{-r}^{(g+t \phi)(v)} \zeta(\Psi(v, w)) d \mathcal{L}^{1} w\right) d \mathcal{L}^{n-1} v .
$$

Then $\Phi(0) \leq \Phi(t)$ whenever $t \in I$ since $D \in \mathbf{n}_{\epsilon}^{l o c}(Z)$. Thus

$$
0=\Phi^{\prime}(0)=\epsilon \int_{V} \frac{\nabla g \bullet \nabla \phi}{\sqrt{1+|\nabla g|^{2}}} d \mathcal{L}^{n}+\int_{V} \zeta\left(\Psi(v, g(v)) \phi(v) d \mathcal{L}^{n} .\right.
$$

That is, $g$ is a weak solution of

$$
-\epsilon \operatorname{div} J^{-1} \nabla g+\zeta \circ \Psi \circ G=0,
$$

where we have set $J=\sqrt{1+|\nabla g|^{2}}$ and $G(v)=(v, g(v))$ for $v \in V$.

Inasmuch as $\partial g$ is Hölder continuous, standard results on regularity of weak solutions of elliptic equations, as found, for example, in [GT, sect. 8.3], imply that $g$ is 
of class $C^{k+2, \mu}$. Since $a$ is an arbitrary point of $M$ we conclude that $M$ is of class $C^{k+2, \mu}$, so $M$ has a second fundamental form. Since $H(a) \bullet \mathbf{n}_{\Gamma}(a)=-\operatorname{div} J^{-1} \nabla g(0)$ we find that (1.7) holds.

Part 2. We now suppose $\zeta$ is continuously differentiable. Let $\Pi, Q, H$ be as in section 4 .

Since $M$ is of class $C^{2}$ by Part 1 there is a map $N: \Omega \rightarrow \mathbb{R}^{n}$ of class $C^{1}$ such that $N\left|M=\mathbf{n}_{\Gamma}\right| M$.

Suppose $\phi \in \mathcal{D}(\Omega)$. Let $X=\phi N$, and let $K ; I ; h_{t}, t \in I ; E_{t}, t \in I ; P$; and $a_{1}$ and $a_{2}$ be as in section 3. Let $Y$ be as in Proposition 3.3. Since $\partial X=(\partial \phi) N+\phi(\partial N)$ we find that

$$
\begin{aligned}
l_{1} & =P \circ \partial(\phi N) \circ P=\phi(P \circ \partial N \circ P), \\
l_{2} & =P^{\perp} \circ \partial(\phi N) \circ P=((\partial \phi) \circ P) N, \\
\operatorname{trace} l_{1} & =\phi(H \bullet N), \\
\operatorname{trace} l_{2}^{*} \circ l_{2} & =|(\partial \phi) \circ P|^{2}, \\
\operatorname{trace} l_{1} \circ l_{1} & =\phi^{2} Q .
\end{aligned}
$$

For each $t \in I$ let

$$
\Phi(t)=\epsilon\left\|\partial\left[E_{t}\right]\right\|(K)+Z\left(E_{t}\right) .
$$

Let $A$ and $B$ be as in Propositions 3.1 and 3.3, respectively, so $\Phi(t)=\epsilon A(t)+B(t)$ for $t \in I$. Since $\Phi(0) \leq \Phi(t)$ for $t \in I$ we have

$$
0 \leq \epsilon A^{\prime \prime}(0)+B^{\prime \prime}(0) .
$$

We have

$$
\begin{aligned}
a_{2} & =\left(\operatorname{trace} l_{1}\right)^{2}+\operatorname{trace}\left(l_{2}^{*} \circ l_{2}-l_{1} \circ l_{1}\right) \\
& =\phi^{2}(H \bullet N)^{2}+|\partial \phi \circ P|^{2}-\phi^{2} Q^{2} \\
& =\phi^{2} \frac{\zeta^{2}}{\epsilon^{2}}+|\partial \phi \circ P|^{2}-\phi^{2} Q^{2} .
\end{aligned}
$$

Making use of (1.7) we obtain

$$
\begin{aligned}
(\zeta Y & +(\nabla \zeta \bullet X) X) \bullet N \\
& =(\zeta(H \bullet(\phi N) \phi N-\nabla \zeta \bullet(\phi N) \phi N) \bullet N \\
& =-\frac{\zeta^{2}}{\epsilon} \phi^{2}+\phi^{2}(\nabla \zeta \bullet N) .
\end{aligned}
$$

So (1.8) now follows from (7.2) and Propositions 3.1 and 3.3.

8. The denoising case revisited. Suppose

(i) $s, \gamma$, and $F$ are as in section 1.8 ;

(ii) $\gamma$ is convex and $\beta$ is as in section 1.8;

(iii) $U$ is an open subset of $\Omega, z \in \mathbb{R}$, and

$$
s(x)=z \quad \text { for } x \in U ;
$$

(iv) $0<y<\infty$ and $\beta$ is continuously differentiable near $y-z$;

(v) $0<\epsilon<\infty$ and $f \in \mathbf{m}_{\epsilon}^{\text {loc }}(F)$; 
(vi) $\Gamma$ is the intersection of $U$ with the interior of the support of $[\{f>y\}]$, and $M$ is the intersection of $U$ with the boundary of $\Gamma$;

(vii) $H$ is the mean curvature vector of $M$, and $Q$ is the square of the length of the second fundamental form of $M$.

From Theorems 1.3 and 1.6 we find that $[\Gamma]=[U \cap\{f>y\}]$, that

$$
H(x)=-\frac{1}{\epsilon} \beta(y-z) \mathbf{n}_{\Gamma}(x) \quad \text { whenever } x \in M,
$$

and that

$$
\int_{M}\left|\nabla_{M} \phi(x)\right|^{2}-\phi(x)^{2} Q(x) d \mathcal{H}^{n-1} x \geq 0
$$

for any $\phi \in \mathcal{D}(\Omega)$, where, for each $x \in M, \nabla_{M} \phi(x)$ is the orthogonal projection of $\nabla \phi(x)$ on $\operatorname{Tan}(M, x)$.

Now suppose $n=2$, let $a \in M$, and let $A$ be the connected component of $a$ in $M$. If $\beta(y-z)=0$, then $A$ is a subset of a straight line. Suppose $\beta(y-z) \neq 0$ and let

$$
R=\frac{\epsilon}{|\beta(y-z)|}
$$

Then $A$ is an arc of a circle of radius $R$. Let $c$ be the center of this circle. Then for each $a \in A$, there is an open subset $G$ of $U$ containing $a$ such that

$$
\Gamma \cap G= \begin{cases}\mathbf{U}^{2}(c, R) \cap G & \text { if } \beta(y-z)<0, \\ \left(\mathbb{R}^{n} \sim \mathbf{U}^{2}(c, R)\right) \cap G & \text { if } \beta(y-z)>0 .\end{cases}
$$

Finally, let $L$ be the length of $A$. Since $Q(x)=1 / R^{2}$ for $x \in M$ we find that

$$
\int_{0}^{L} \phi^{\prime}(\sigma)^{2}-\frac{1}{R^{2}} \phi(\sigma)^{2} d \mathcal{L}^{1} \sigma \geq 0
$$

for all continuously differentiable $\phi:[0, L] \rightarrow \mathbb{R}$ which are differentiable on $(0, L)$ and which vanish at 0 and $L$. Letting $\phi(\sigma)=\sin (\pi \sigma / L)$ for $\sigma \in[0, L]$ we infer that

$$
L \leq \pi R .
$$

\section{Some results for functionals on sets.}

9.1. Proof of Theorem 1.7. We begin with a simple lemma.

Lemma 9.1. Suppose $A$ is a nested sequence in $\mathbf{n}_{\epsilon}^{\text {loc }}\left(N_{S}\right)$. Then $\cap_{\nu=1}^{\infty} A_{\nu} \in$ $\mathbf{n}_{\epsilon}^{\text {loc }}\left(N_{S}\right)$ and, provided $\mathcal{L}^{n}\left(\cup_{\nu=1}^{\infty} A_{\nu}\right)<\infty, \cup_{\nu=1}^{\infty} \in \mathbf{n}_{\epsilon}^{\text {loc }}\left(N_{S}\right)$.

We leave to the reader the straightforward proof making use of (2.1) and cutoff arguments like those used in the proof of Theorem 5.2.

Let

$$
F(f)=\int_{\Omega}\left|f-1_{S}\right| d \mathcal{L}^{n} \quad \text { for } f \in \mathcal{F}(\Omega) .
$$

For each $y \in \mathbb{R}$ let $U_{y}$ be as in Theorem 1.5. Recall from section 1.9 that

$$
U_{y}= \begin{cases}0 & \text { if } 1 \leq y<\infty \\ \widehat{N_{S}} & \text { if } 0<y<1\end{cases}
$$


Suppose $A, B \in \mathcal{A}$ and $0<a<b<c<1$. Let

$$
G=(A \times(0, b)) \cup(B \times(b, 1)) \in \mathcal{G}(\Omega) .
$$

Then $\{x:(x, y) \in G\} \in \mathbf{n}_{\epsilon}^{\text {loc }}\left(U_{y}\right)$ whenever $0<y<\infty$, so $G^{\downarrow} \in \mathbf{m}_{\epsilon}^{\text {loc }}(F)$ by Theorem 1.4. From Theorem 1.3 we infer that $A \cup B=\left\{G^{\downarrow}>a\right\} \in \mathbf{n}_{\epsilon}^{\text {loc }}\left(U_{a}\right)$ and $A \cap B$ $=\left\{G^{\downarrow}>c\right\} \in \mathbf{n}_{\epsilon}^{l o c}\left(U_{c}\right)$, so $A \cup B$ and $A \cap B$ belong to $\mathbf{n}_{\epsilon}^{l o c}\left(N_{S}\right)$.

It follows that if $\mathcal{F}$ is a finite subfamily of $\mathcal{A}$, then $\cup \mathcal{F}$ and $\cap \mathcal{F}$ belong to $\mathbf{n}_{\epsilon}^{\text {loc }}\left(N_{S}\right)$.

Let $B$ be a sequence in $\mathcal{A}$ such that

$$
\lim _{\nu \rightarrow \infty} \mathcal{L}^{n}\left(B_{\nu}\right)=\inf \left\{\mathcal{L}^{n}(A): A \in \mathcal{A}\right\} .
$$

Since each of $\cap_{\nu=1}^{N} B_{\nu}$ belongs to $\mathbf{n}_{\epsilon}^{\text {loc }}\left(N_{S}\right)$ we infer from the preceding lemma that $C=\cap_{\nu=1}^{\infty} B_{\nu} \in \mathbf{n}_{\epsilon}^{\text {loc }}\left(N_{S}\right)$. It is clear that $\mathcal{L}^{n}(C \sim \cap \mathcal{A})=0$, so $\cap \mathcal{A} \in \mathbf{n}_{\epsilon}^{\text {loc }}\left(N_{S}\right)$.

Let us now assume $\mathcal{L}^{n}(\cup \mathcal{A})<\infty$. Let $D$ be a sequence in $\mathcal{A}$ such that

$$
\lim _{\nu \rightarrow \infty} \mathcal{L}^{n}\left(D_{\nu}\right)=\sup \left\{\mathcal{L}^{n}(A): A \in \mathcal{A}\right\} .
$$

Since each of $\cup_{\nu=1}^{N} D_{\nu}$ belongs to $\mathbf{n}_{\epsilon}^{\text {loc }}\left(N_{S}\right)$ we infer from the preceding lemma that $E=\cup_{\nu=1}^{\infty} D_{\nu} \in \mathbf{n}_{\epsilon}^{l o c}\left(N_{S}\right)$. It is clear that $\mathcal{L}^{n}(\cup \mathcal{A} \sim E)=0$, so $\cup \mathcal{A} \in \mathbf{n}_{\epsilon}^{\text {loc }}\left(N_{S}\right)$.

9.2. A comparison principle. The following proposition and its proof were suggested by a similar result found in [CA1] in a different context.

Proposition 9.1. Suppose $M, N \in \mathbf{M}(\Omega), M$ and $N$ are local, $0<\epsilon<\infty$, $D \in \mathbf{n}_{\epsilon}^{l o c}(M), E \in \mathbf{n}_{\epsilon}^{\text {loc }}(N)$, and $\mathbf{s p t}[D \cup E]$ is compact. Then

$$
\hat{N}(E \sim D) \leq \hat{M}(E \sim D) .
$$

In particular, if

$$
\hat{M}(G)<\hat{N}(G) \quad \text { whenever } G \in \mathcal{M}(\Omega) \text { and } \mathcal{L}^{n}(G)>0,
$$

then

$$
\mathcal{L}^{n}(E \sim D)=0
$$

Proof. Without loss of generality we may assume $M=\hat{M}$ and $N=\hat{N}$. Since spt $[D] \cup \operatorname{spt}[E] \subset \mathbf{s p t}[D \cup E]$ we have

$$
\epsilon \mathbf{M}(\partial[D])+M(D) \leq \epsilon \mathbf{M}(\partial[D \cup E])+M(D \cup E)
$$

and

$$
\epsilon \mathbf{M}(\partial[E])+N(E) \leq \epsilon \mathbf{M}(\partial[D \cap E])+N(D \cap E) .
$$

Also,

$$
\begin{aligned}
\mathbf{M}(\partial[ & {[D \cup E])+\mathbf{M}(\partial[D \cap E]) } \\
& =\int_{0}^{1} \mathbf{M}\left(\partial\left[\left\{1_{D}+1_{E}>y\right\}\right] d \mathcal{L}^{1} y+\int_{1}^{2} \mathbf{M}\left(\partial\left[\left\{1_{D}+1_{E}>y\right\}\right] d \mathcal{L}^{1} y\right.\right. \\
& =\mathbf{M}\left(\partial\left[1_{D}+1_{E}\right]\right) \\
& \leq \mathbf{M}(\partial[D])+\mathbf{M}(\partial[E]) .
\end{aligned}
$$


Since $M$ and $N$ are local it follows that

$$
\begin{aligned}
& \epsilon(\mathbf{M}(\partial[D])+\mathbf{M}(\partial[E]))+M(D \sim E)+M(D \cap E)+N(E \sim D)+N(E \cap D) \\
= & \epsilon(\mathbf{M}(\partial[D])+\mathbf{M}(\partial[E]))+M(D)+N(E) \\
\leq & \epsilon(\mathbf{M}(\partial[D \cup E])+\mathbf{M}(\partial[D \cap E]))+M(D \cup E)+N(D \cap E) \\
\leq & \epsilon(\mathbf{M}(\partial[D])+\mathbf{M}(\partial[E]))+M(D \cup E)+N(D \cap E) \\
= & \epsilon(\mathbf{M}(\partial[D])+\mathbf{M}(\partial[E]))+M(D \sim E)+M(D \cap E)+M(E \sim D)+N(E \cap D) .
\end{aligned}
$$

9.3. Proof of Theorem 1.5. Suppose $0<y<z<\infty$. Since $F$ is strictly convex we have

$$
\beta(x, y)<\beta(x, z) \text { for } \mathcal{L}^{n} \text { almost all } x \in \Omega .
$$

This in turn implies that $U_{y}(D)<U_{z}(D)$ whenever $D \in \mathcal{M}(\Omega)$. Applying Proposition 9.1 with $M, N$ there equal to $U_{y}, U_{z}$ and $D, E$ there equal to $\{f>y\},\{x:(x, z) \in G\}$ and $\{x:(x, y) \in G\},\{f>z\}$, respectively, we infer that

$$
\mathcal{L}^{n}(\{x:(x, z) \in G\} \sim\{f>y\})=0
$$

and

$$
\mathcal{L}^{n}(\{f>z\} \sim\{x:(x, y) \in G\})=0 .
$$

Suppose $0<w<\infty$. Letting $z=w$ and $y \uparrow w$ in (i) we find that

$$
\mathcal{L}^{n}(\{x:(x, w) \in G\} \sim\{f \geq w\})=0 .
$$

Letting $y=w$ and $z \downarrow w$ in (ii) we find that

$$
\mathcal{L}^{n}(\{f>w\} \sim\{x:(x, w) \in G\})=0 .
$$

Since $\mathcal{L}^{n}(\{f=w\})=0$ for all but countably many $w \in(0, \infty)$ we may use Tonelli's theorem to complete the proof.

10. Two useful theorems in the denoising case. We suppose throughout this subsection that $\gamma: \mathbb{R} \rightarrow \mathbb{R}, \gamma$ is locally Lipschitzian, $\gamma$ is decreasing on $(-\infty, 0)$, and $\gamma$ is increasing on $(0, \infty)$. We let

$$
F(f)=\int_{\Omega} \gamma(f(x)-s(x)) d \mathcal{L}^{n} x \quad \text { whenever } f \in \mathcal{F}(\Omega) .
$$

10.1. A simple maximum principle.

Proposition 10.1. Suppose $0<\epsilon<\infty, f \in \mathbf{m}_{\epsilon}^{\text {loc }}(F)$, and

$$
u=\inf \left\{\left\|1_{\Omega \sim K} f\right\|_{\mathbf{L}_{\infty}(\Omega)}: K \text { is a compact subset of } \Omega\right\} .
$$

Then $\|f\|_{\mathbf{L}_{\infty}(\Omega)} \leq u \vee\|s\|_{\mathbf{L}_{\infty}(\Omega)}$.

Remark 10.1. It follows from Corollary 5.1 that $u=0$ if $\Omega=\mathbb{R}^{n}$.

Proof. Suppose $u \vee\|s\|_{\mathbf{L}_{\infty}(\Omega)}<M<\infty$. Then $K=\operatorname{spt}[f-f \wedge M]$ is a compact subset of $\Omega$, so

$$
\begin{aligned}
\int_{\{f>M\}} & \gamma(f(x)-s(x))-\gamma(M-s(x)) d \mathcal{L}^{n} x \\
& =F(f)-F(f \wedge M) \\
& \leq \epsilon(\|\partial[f \wedge M]\|(K)-\|\partial[f]\|(K)) \\
& =-\int_{M}^{\infty}\|\partial[\{f>y\}]\|(K) d \mathcal{L}^{1} y \\
& \leq 0 .
\end{aligned}
$$


If $f(x)>M>s(x)$, then $f(x)-s(x)>M-s(x)>0$, so that $\gamma(f(x)-s(x))-\gamma(M-$ $s(x))>0$. Owing to the arbitrariness of $M$ we find that the proposition holds.

Theorem 10.1. Suppose $\Omega=\mathbb{R}^{n}, 0<\epsilon<\infty, f \in \mathbf{m}_{\epsilon}^{\text {loc }}(F)$, and, for each $y \in(0, \infty)$,

$$
C(y) \text { equals the closed convex hull of } \mathbf{s p t}[\{s>y\}] \text {. }
$$

Then

$$
\text { spt }[\{f>y\}] \subset C(y) \quad \text { whenever } 0<y<\infty .
$$

Proof. Suppose $0<b<\infty$. Let $g_{b}=f 1_{\{f<b\}}+b 1_{\{f \geq b\} \sim C(b)}+f 1_{\{f \geq b\} \cap C(b)}$ and note that

$$
\left\{g_{b}>y\right\}=\left\{\begin{array}{ll}
\{f>y\} & \text { if } y \leq b, \\
\{f>y\} \cap C(b) & \text { if } y>b
\end{array} \quad \text { whenever } y \in \mathbb{R} .\right.
$$

It follows from (2.12) that $\mathbf{M}\left(\partial\left[\left\{g_{b}>y\right\}\right]\right) \leq \mathbf{M}(\partial[\{f>y\}])$ whenever $y \in \mathbb{R}$.

Let $K_{b}=\operatorname{spt}\left[f-g_{b}\right]$. Since $\left\{f-g_{b} \neq 0\right\} \subset\{f>b\}$ we infer from Theorems 1.1 and 5.4(v) that $K_{b}$ is compact. Since $f \in \mathbf{m}_{\epsilon}^{\text {loc }}(F)$ we infer with the help of (5.1) that

$$
\begin{aligned}
\int_{\{f>b\} \sim C(b)} & \gamma(f(x)-s(x))-\gamma(b-s(x)) d \mathcal{L}^{n} x \\
& =F(f)-F\left(g_{b}\right) \\
& \leq \epsilon\left(\left\|\partial\left[g_{b}\right]\right\|\left(K_{b}\right)-\|\partial[f]\|\left(K_{b}\right)\right) \\
& =\epsilon \int_{b}^{\infty}\left\|\partial\left[\left\{g_{b}>y\right\}\right]\right\|\left(K_{b}\right)-\|\partial[\{f>y\}]\|\left(K_{b}\right) d \mathcal{L}^{1} y \\
& \leq 0
\end{aligned}
$$

which implies $\mathcal{L}^{n}(\{f>b\} \sim C(b))=0$.

\subsubsection{Convex containment.}

Proposition 10.2. Suppose $M \in \mathbf{M}\left(\mathbb{R}^{n}\right), M$ is local, $C$ is a closed convex subset of $\mathbb{R}^{n}$, and

$$
M(E) \geq M(\emptyset) \quad \text { whenever } E \in \mathcal{M}\left(\mathbb{R}^{n}\right) \text { and } \quad \mathcal{L}^{n}(E \cap C)=0 .
$$

Then $\mathbf{s p t}[D]$ is a compact subset of $C$ whenever $D \in \mathbf{n}_{\epsilon}^{\text {loc }}(M)$.

Remark 10.2. Evidently, (10.1) is equivalent to the statement that $\mu(x) \geq 0$ for $\mathcal{L}^{n}$ almost all $x \in \mathbb{R}^{n} \sim C$, where $\mu$ is as in Proposition 1.3.

Proof. Suppose $D \in \mathbf{n}_{\epsilon}^{\text {loc }}(M)$. It follows from Proposition 1.2 and Theorem 5.4(iv) that $\mathbf{s p t}[D]$ is compact. From (2.12) we find that

$$
\mathbf{M}(\partial[C \cap D]) \leq \mathbf{M}(\partial[D]) .
$$

Moreover, as $M$ is local and $D \in \mathbf{n}_{\epsilon}^{l o c}(M)$,

$$
\epsilon(\mathbf{M}(\partial[D])-\mathbf{M}(\partial[D \cap C])) \leq M(D \cap C)-M(D)=M(\emptyset)-M(D \sim C) \leq 0 .
$$

Thus $\mathbf{M}(\partial[C \cap D])=\mathbf{M}(\partial[D])$, so the theorem now follows from (2.12). 
11. Some examples. Let

$$
S=[-1,1] \times[-1,1] \in \mathcal{M}\left(\mathbb{R}^{2}\right),
$$

suppose $1 \leq p<\infty$, and let

$$
F(g)=\frac{1}{p} \int\left|g-1_{S}\right|^{p} d \mathcal{L}^{2} \quad \text { whenever } g \in \mathcal{M}\left(\mathbb{R}^{2}\right) .
$$

We will determine $\mathbf{m}_{\epsilon}^{\text {loc }}(F), 0<\epsilon<\infty$.

11.1. The sets $\boldsymbol{A}_{\boldsymbol{i}, \boldsymbol{r}}$. For each $r \in(0,1]$ let

$$
A_{0, r}=\{(1-r, 1-r)+r\{(\cos \theta, \sin \theta): 0 \leq \theta \leq \pi / 2\},
$$

let $A_{i, r}, i=1,2,3$, be a counterclockwise rotation about the origin of $A_{0, r}$ by $i \pi / 2$, and let

$$
C(r)
$$

be the convex hull of $\cup_{i=0}^{3} A_{i, r}$.

Theorem 11.1. Suppose $0<\epsilon<\infty$ and

$$
T=\left\{[f]: f \in \mathbf{m}_{\epsilon}^{l o c}(F)\right\} .
$$

If $(1+\sqrt{\pi} / 2) \epsilon>1$, then

$$
T=\{0\} .
$$

If $(1+\sqrt{\pi} / 2) \epsilon=1$ and $p=1$, then

$$
T=\left\{t\left[1_{C(\epsilon)}\right]: 0 \leq t \leq 1\right\} .
$$

If $(1+\sqrt{\pi} / 2) \epsilon<1$ and $p=1$, then

$$
T=\left\{\left[1_{C(\epsilon)]}\right\} .\right.
$$

If $(1+\sqrt{\pi} / 2) \epsilon=1$ and $p>1$, then

$$
T=\{0\} .
$$

If $(1+\sqrt{\pi} / 2) \epsilon<1$ and $p>1$, then

$$
T=\left\{\left[G^{\downarrow}\right]\right\},
$$

where

$$
Y=1-(1+\sqrt{\pi} / 2) \epsilon)^{1 /(p-1)}
$$

and

$$
G=\left\{(x, y): 0<y<Y \text { and } x \in C\left(\frac{\epsilon}{(1-y)^{p-1}}\right)\right\} \in \mathcal{G}\left(\mathbb{R}^{2}\right) .
$$

Proof. For each $y \in(0, \infty)$ let

$$
Q_{y}=\left\{[D]: D \in \mathbf{n}_{\epsilon}^{l o c}\left(U_{y}\right)\right\}
$$


where $U_{y}$ is as in Theorem 1.5.

Using (1.9) we find that $U_{y}(E)>0$ whenever $1<y<\infty, E \in \mathcal{M}\left(\mathbb{R}^{n}\right)$, and $\mathcal{L}^{2}(E)>0$; since $U_{y}(\emptyset)=0$ we find that

$$
Q_{y}=\{0\} \quad \text { if } 1<y<\infty .
$$

Suppose $0<y<1$, let

$$
Z=\left\{\begin{array}{ll}
1 & \text { if } p=1, \\
(1-y)^{p-1} & \text { if } p>1,
\end{array} \quad \text { and let } \quad R=\frac{\epsilon}{Z}\right.
$$

Suppose $R \leq 1$ and let

$$
I=\left(U_{y}\right)_{\epsilon}(C(R))=\epsilon \mathbf{M}(\partial[C(R)])+U_{y}(C(R)) .
$$

We have

$$
\epsilon \mathbf{M}(\partial[C(R)])=\epsilon(4(2-2 R)+2 \pi R)
$$

and

$$
U_{y}(C(R))=-Z \mathcal{L}^{2}(C(R))=-Z\left(4-(4-\pi) R^{2}\right),
$$

so

$$
\begin{aligned}
I & =\epsilon(4(2-2 R)+2 \pi R)-Z\left(4-(4-\pi) R^{2}\right) \\
& =\frac{-4 Z^{2}+8 \epsilon Z+(\pi-4) \epsilon^{2}}{Z} \\
& =-4 \frac{(Z-(1+\sqrt{\pi} / 2) \epsilon)(Z-(1-\sqrt{\pi} / 2) \epsilon)}{Z} .
\end{aligned}
$$

Since $R \leq 1$ we have

$$
Z=\epsilon / R \geq \epsilon>(1-\sqrt{\pi} / 2) \epsilon
$$

Thus

$$
I\left\{\begin{aligned}
<0=U_{y}(\emptyset) & \Leftrightarrow Z>(1+\sqrt{\pi} / 2) \epsilon, \\
=0=U_{y}(\emptyset) & \Leftrightarrow Z=(1+\sqrt{\pi} / 2) \epsilon, \\
>0=U_{y}(\emptyset) & \Leftrightarrow Z<(1+\sqrt{\pi} / 2) \epsilon .
\end{aligned}\right.
$$

Suppose $D \in \mathbf{n}_{\epsilon}^{l o c}\left(U_{y}\right),[D] \neq 0$, and $D=\mathbf{s p t}[D]$. We claim that

$$
R \leq 1 \quad \text { and } \quad D=C(R) .
$$

From Proposition 10.2 we infer that $D \subset S$. Let $U$ equal the interior of $S$ and let $M=U \cap$ bdry $D$. Then $U \cap M \neq \emptyset$ since otherwise we would have $D=S$, in which case $M$ would have corners, which is incompatible with Theorem 1.2. Let $A$ be a connected component of $M$. We infer from section 8 that $A$ is an arc of a circle of radius $R$, the length of which does not exceed $\pi R$. Because $D$ can have no corners we find that $A$ meets the interior of the boundary of $S$ tangentially. Thus (11.1) holds.

The theorem now follows from Theorems 1.3 and 1.4. 
Acknowledgments. It is a pleasure to thank Kevin Vixie for acquainting me with this area of research. In the course of carrying out this work I profited from conversations with Kevin Vixie and Selim Esedoğlu and benefited from the support of Los Alamos National Laboratory.

\section{REFERENCES}

[Alli] S. S. Alliney, Digital filters as absolute norm regularizers, IEEE Trans. Signal Process., 40 (1992), pp. 1548-1562.

[AW1] W. K. Allard, The first variation of a varifold, Ann. Math., 95 (1972), pp. 417-491.

[AW2] W. K. Allard, Total variation regularization for image denoising; II. Examples, in preparation.

[BDG] E. Bombieri, E. DeGiorgi, and E. Giusti, Minimal cones and the Bernstein problem, Invent. Math., 7 (1969), pp. 243-268.

[CA1] A. Chambolle, Total Variation Minimization and a Class Of Binary MRF Models, preprint CMAP 578, Centre de Mathématiques Appliquées, École Polytechnique, Palaiseau, France; available online from http://www.cmap.polytechnique.fr/preprint/repository/ 578.pdf; this version varies a bit from that in Energy Minimization Methods in Computer Vision and Pattern Recognition: 5th International Workshop, EMMCVPR 2005, Lecture Notes in Comput. Sci. 3757, Springer-Verlag, Berlin, 2005, pp. 136-152.

[CA2] A. Chambolle, An algorithm for mean curvature motion, Interfaces Free Bound., 6 (2004), pp. $195-218$

[CE] T. F. Chan And S. Esedō̄Lu, Aspects of total variation regularized $L^{1}$ function approximation, SIAM J. Appl. Math., 65 (2005), pp. 1817-1837.

[FE] H. Federer, Geometric Measure Theory, Grundlehren Math. Wiss. 153, Springer-Verlag, New York, 1969.

[GT] D. Gilbarg and N. S. Trudinger, Elliptic Partial Differential Equations of Second Order, Grundlehren Math. Wiss. 224, Springer-Verlag, New York, 1977.

[M] U. MASSARI, Esistenza e regolaritá delle ipersuperficie di curvatura media assegnata in $\mathbb{R}^{n}$, Arch. Ration. Mech. Anal., 55 (1974), pp. 357-382.

[ROF] L. Rudin, S. Osher, AND E. FAtemi, Nonlinear total variation based noise removal algorithms, Phys. D, 60 (1992), pp. 259-268. 